\title{
Trends of mortality attributable to child and maternal undernutrition, overweight/obesity and dietary risk factors of non- communicable diseases in sub-Saharan Africa, 1990-2015: findings from the Global Burden of Disease Study 2015
}

\author{
Yohannes Adama Melaku 1,2,* Tiffany K Gill², Anne W Taylor ${ }^{2}$, Sarah L Appleton ${ }^{3,4}$, \\ David Gonzalez-Chica ${ }^{2}$, Robert Adams ${ }^{3}$, Tom Achoki ${ }^{5}$, Zumin Shi ${ }^{2,6}$ and \\ Andre Renzaho7,8 \\ ${ }^{1}$ Department of Human Nutrition, Institute of Public Health, University of Gondar, Gondar, Ethiopia: ${ }^{2}$ Adelaide \\ Medical School, University of Adelaide, SAHMRI, North Terrace, Adelaide, SA 5000, Australia: ${ }^{3}$ The Health \\ Observatory, Discipline of Medicine, University of Adelaide, Queen Elizabeth Hospital Campus, Woodville, SA, \\ Australia: ${ }^{4}$ Freemason's Centre for Men's Health, Discipline of Medicine, University of Adelaide, Adelaide, SA, \\ Australia: ${ }^{5}$ Institute for Health Metrics and Evaluation, University of Washington, Seattle, WA, USA: ${ }^{6}$ Human \\ Nutrition Department, College of Health Sciences, Qatar University, Doha, Qatar: ${ }^{7}$ Humanitarian and Development \\ Research Initiative, School of Social Sciences and Psychology, Western Sydney University, Penrith, NSW, Australia: \\ ${ }^{8}$ School of Public Health and Preventive Medicine, Monash University, Melbourne, VIC, Australia
}

Submitted 28 July 2018: Final revision received 25 September 2018: Accepted 1 October 2018: First published online 4 December 2018

\begin{abstract}
Objective: To assess trends of mortality attributable to child and maternal undernutrition (CMU), overweight/obesity and dietary risks of non-communicable diseases (NCD) in sub-Saharan Africa (SSA) using data from the Global Burden of Disease (GBD) Study 2015.

Design: For each risk factor, a systematic review of data was used to compute the exposure level and the effect size. A Bayesian hierarchical meta-regression analysis was used to estimate the exposure level of the risk factors by age, sex, geography and year. The burden of all-cause mortality attributable to CMU, fourteen dietary risk factors (eight diets, five nutrients and fibre intake) and overweight/obesity was estimated.

Setting: Sub-Saharan Africa.

Participants: All age groups and both sexes.

Results: In 2015, CMU, overweight/obesity and dietary risks of NCD accounted for 826204 (95\% uncertainty interval (UI) 737 346, 923 789), 266768 (95\% UI 189051 , $353096)$ and 558578 (95\% UI 453433, 680 197) deaths, respectively, representing $10 \cdot 3 \%$ (95\% UI $9 \cdot 1,11 \cdot 6 \%$ ), $3 \cdot 3 \%$ (95\% UI $2 \cdot 4,4 \cdot 4 \%$ ) and $7 \cdot 0 \%$ (95\% UI $5 \cdot 8$, $8.3 \%)$ of all-cause mortality. While the age-standardized proportion of all-cause mortality accounted for by CMU decreased by 55.2\% between 1990 and 2015 in SSA, it increased by 63.3 and $17 \cdot 2 \%$ for overweight/obesity and dietary risks of NCD, respectively.

Conclusions: The increasing burden of diet- and obesity-related diseases and the reduction of mortality attributable to CMU indicate that SSA is undergoing a rapid nutritional transition. To tackle the impact in SSA, interventions and international development agendas should also target dietary risks associated with NCD and overweight/obesity.
\end{abstract}

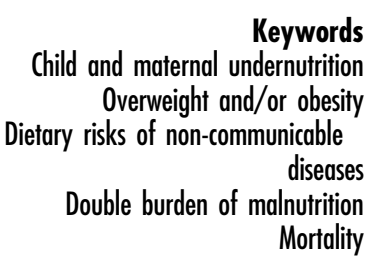

Long-standing food and nutrition insecurity in sub-Saharan Africa (SSA) has caused a high burden of communicable, maternal, neonatal and nutritional $(\mathrm{CMNN})$ diseases $^{(1,2)}$ and economic loss ${ }^{(3)}$. In recent years, however, the unprecedented rising burden of non-communicable diseases (NCD) ${ }^{(4)}$ associated with increased metabolic ${ }^{(5)}$ and behavioural risks ${ }^{(6,7)}$ is a major public health concern. Particularly, dietary risk factors and overweight/obesity 
are the major contributors to the rising burden of NCD in the region ${ }^{(6,8)}$. Available data suggest that the consumption of processed foods in $\mathrm{SSA}^{(9)}$ and the prevalence of obesity $^{(10)}$ in the region have increased significantly over the past two decades. Obesity rates ${ }^{(11)}$ have increased while rates of undernutrition, especially stunting among children, have stagnated ${ }^{(12)}$, hence translating into the double burden of malnutrition, a phenomenon characterized by the coexistence of undernutrition, overweight/obesity and diet-related $\mathrm{NCD}^{(13)}$. The majority of intervention efforts in SSA still focus on addressing child and maternal undernutrition (CMU). Investigating the impact of the double burden is imperative to design appropriate interventions.

As part of the Global Burden of Disease (GBD) studies, a recent analysis demonstrated the increasing prevalence of overweight/obesity and related burden of disease at the global level. The study reported that 4 million deaths were attributable to excess body weight worldwide in $2015^{(11)}$. Another study also found different levels (prevalence and rate) of nutrition-related disorders and diseases (such as low birth weight, stunting, underweight, infant mortality rate, overweight/obesity and NCD mortality) in forty SSA countries $^{(14)}$. However, these studies did not comprehensively look at the impact of the double burden because they did not include important risk factors and/or did not focus on SSA. In addition, although studies have shown the presence of the double burden of malnutrition (high rates of undernutrition, increasing prevalence of overweight/obesity and diet-related NCD) in SSA ${ }^{(15-17)}$, the proportion and trend of mortality attributable to this phenomenon have not been investigated at different levels (SSA, SSA sub-regions and countries). One of the challenges in SSA, where the health infrastructure is poor, is the limited epidemiological data that are readily or reliably available $^{(18)}$, particularly on overweight/obesity and dietary risk factors of NCD. In the current paper, using the GBD 2015 data $^{(19)}$, we aimed to assess the impact of the double burden of malnutrition, specifically by reporting and comparing estimates and mortality trends associated with CMU, overweight/obesity and dietary risk factors of NCD in SSA between 1990 and 2015. We examined the patterns of attributable mortality by year, sex and age across the SSA sub-regions and countries.

\section{Methods}

\section{Study overview}

GBD is a large epidemiological study that measures and evaluates health status and its determinants across the global population. It includes assessments of diseases, injuries, sequelae and risk factors. In particular, the 2015 GBD risk factors study covers seventy-nine risk factors of health across seven regions, twenty-one sub-regions and 195 countries $^{(2)}$. The study uses a comparative risk assessment approach, which is a causal web and hierarchy of risk factors that enables the quantification of risk factors and their impact on health at different levels ${ }^{(20)}$. In the framework, risk factors are organized in four hierarchies. The first hierarchy includes three categories of risk factor clusters: behavioural (including CMU and dietary risks of NCD), environmental/occupational and metabolic (including overweight/obesity). The second (seventeen risk factors) and third (forty-four risk factors) levels contain both granular and cluster risks from within the first level. At the last level (seventy-nine risk factors), granular risk factors are included ${ }^{(2)}$. A detailed description of the GBD 2015 risk factors study methods has been published elsewhere $^{(2,21)}$.

A summary framework of the risk factors that were included in the current study by each of the hierarchies is depicted in Fig. 1. In the current study, we used the GBD databases $^{(19)}$ to specifically examine the mortality burden attributable to twenty-three behavioural and metabolic risk factors (level 4), which were under CMU and the dietary risks of NCD, including overweight/obesity. The current study focused on SSA which covers five sub-regions (Northern, Eastern, Western, Southern and Central SSA) and fifty-four countries. Assignment of countries in the sub-regions was based on the GBD geographical classifications. We highlight methods specific to the current study in the following sections.

\section{Selection of risk factors}

Using the World Cancer Research Fund grading system, risk-disease pairs with convincing or probable evidence were included in the GBD 2015 risk factors study ${ }^{(2,22)}$. CMU included suboptimal breast-feeding (non-exclusive (receiving predominant, partial or no breast-feeding in children younger than 6 months) and discontinued breastfeeding (not able to receive breast milk in children aged 6-23 months)), child undernutrition (childhood stunting, underweight and wasting), and $\mathrm{Fe}, \mathrm{Zn}$ and vitamin $\mathrm{A}$ deficiencies. Dietary risks of NCD comprised fourteen factors, including eight diets (diets low in fruits, vegetables, milk, nuts and seeds, and whole grains; diets high in processed meat, red meat and sugar-sweetened beverages), five nutrients (diets low in seafood $n$ - 3 fatty acids, $\mathrm{Ca}$ and PUFA; diets high in Na and SFA) and fibre intake (Fig. 1). A BMI of $25.0 \mathrm{~kg} / \mathrm{m}^{2}$ or more was considered the cut-off for being at risk of different diseases including cancer, CVD and musculoskeletal disorders ${ }^{(2)}$. Specific methods of incidence, prevalence and burden of disease associated with overweight/obesity estimates have been published elsewhere ${ }^{(11)}$. The list, definition and global data representativeness index (calculated by dividing the number of countries with risk factors data by the total number of countries in the world and multiplying by 100) of the risk factors are provided in the online supplementary material, Supplemental Table 1. 


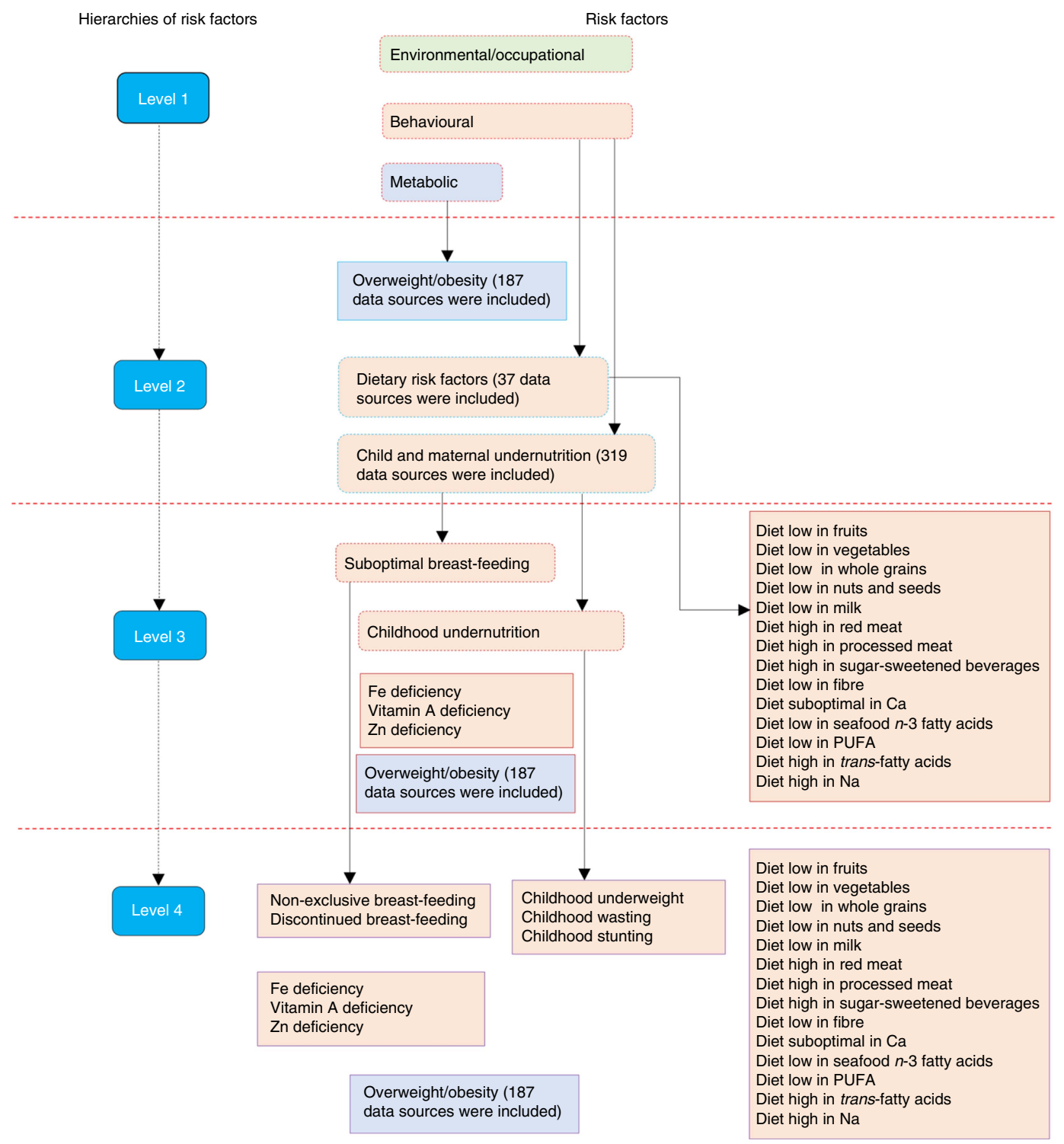

Fig. 1 (colour online) Hierarchies of included risk factors (adapted from the Global Burden of Disease (GBD) 2015 risk factors study ${ }^{(2)}$. The figure depicts a summary of child and maternal undernutrition, dietary risk factors of non-communicable diseases and overweight/obesity as risk factors of mortality; more detailed methods can be accessed elsewhere ${ }^{(2)}$

\section{Data sources and exposure levels}

For each risk factor, the literature was systematically searched to identify studies that could potentially provide exposure levels. In the GBD 2015 risk factors study, a total of 543 data sources were included to determine the exposure levels of CMU ( $n$ 319), overweight/obesity ( $n$ 187) and dietary risk factors of NCD ( $n$ 37) in SSA (Fig. 1). In the online supplementary material, Supplemental Tables 2, 3 and 4, we provide the lists of these data sources. Data sources by sub-regions and countries can also be accessed on the Global Health Data Exchange (http:// ghdx.healthdata.org/gbd-2015/data-input-sources).

For CMU, data were mainly obtained from demographic and health surveys, microdata of surveys, reproductive and health surveys, multiple indicator cluster surveys and living standards measurement surveys, scientific literature, FAO food balance sheets, and UNICEF and WHO databases. Data from the National Center for Health Statistics/ WHO (the NCHS reference) were also used ${ }^{(2)}$.

Multiple data sources, including FAO food balance sheets, household budget surveys, FFQ, and single as well as multiple diet recall surveys were used to estimate exposure levels of dietary risks for NCD. For trans-fatty acids, availability of packaged foods containing partially hydrogenated vegetable oil was used. Data of urinary $\mathrm{Na}$ and sugar-sweetened beverages were not adjusted to $8368 \mathrm{~kJ} / \mathrm{d}(2000 \mathrm{kcal} / \mathrm{d})$, unlike other dietary risk factors. For overweight/obesity, data were collated from surveys, longitudinal studies, published literature which provided both measured or self-reported height and weight, and 
national or sub-national estimates of BMI, overweight and obesity $^{(2)}$.

Two modelling strategies were used to estimate the exposure levels for the risk factors: a spatiotemporal Gaussian process regression model (ST-GPR) and a Bayesian meta-regression model (DisMod-MR 2.1). These are mixed-effect models that borrow information across geographic location, age, sex and time. These approaches allow for the pooling of data from different sources and for adjustment of bias. Informative covariates are also able to be incorporated to assist in the predictions for locations in time where there was an absence of data. Adjustments were made including age-sex splitting, adding study-level covariates and bias correction for all risk factors ${ }^{(2,23,24)}$.

ST-GPR is a modelling strategy that helps to estimate magnitude of health problems while using multiple and noisy data and helps to interpolate non-linear trends across time and geography without an assumption that the feature of data follows a definitive functional form. ST-GPR assumes a Gaussian process defined by mean and covariance functions ${ }^{(24,25)}$. DisMod-MR 2.1 is a computational engine designed to combine data using Bayesian statistical methods. It has a capacity to organize data at each geographic and analytical level. The analysis was performed at five hierarchies: global, super-region, region, country and sub-national (where applicable) ${ }^{(23,26,27)}$.

For the dietary risks for NCD, first, ST-GPR was applied using the FAO data to model the availability of nutrients and foods. Then, DisMod-MR 2.1 was used to model the intake of each food group and nutrient and to account for study-level covariates (see online supplementary material, Supplemental Table 5). DisMod's ODE solver was utilized to generate a global age trend based on the diet recall data. This age trend was then applied to the FAO results to produce age-specific estimates of food and nutrient availability. Detailed methods are published elsewhere ${ }^{(2,28)}$

\section{Relative risks}

Relative risks of risk-disease pairs were obtained from meta-analyses of prospective observational studies or randomized controlled trials. For non-exclusive breastfeeding, a relative risk was determined using the associations with lower respiratory tract infection and diarrhoea. Discontinued breast-feeding was paired with diarrhoea only. The relative risks of wasting, stunting and underweight were adjusted using an algorithm that accounts for covariance between the three undernutrition indicators. Relative risks for $\mathrm{Zn}$ deficiency were estimated based on the results of clinical trials. The data sources for the relative risks have been described elsewhere ${ }^{(2)}$.

For dietary data, relative risks were obtained from most recent meta-analyses of prospective observational studies, and if available, from randomized controlled trials. Metabolic mediators (BMI, total serum cholesterol, fasting plasma glucose and systolic blood pressure) were considered in the calculation (see online supplementary material, Supplemental Table 5). Due to scarce (inconclusive) evidence, for sugar-sweetened beverages and $\mathrm{Na}$, relative risks were estimated using BMI and systolic blood pressure, respectively. The relative risk of change in BMI for each disease endpoint was obtained from meta-analyses, and if available, pooled analyses of prospective observational studies ${ }^{(2)}$.

\section{Attributable mortality and uncertainties}

The proportion of mortality that could have been prevented if the exposure level of a risk factor had been sustained at the level associated with the lowest risk was calculated. The level of exposure that is associated with the lowest risk is called the theoretical minimum risk exposure level (TMREL). A 20\% uncertainty range below and above the TMREL was considered as an interval associated with the lowest risk and used in determining the burden of disease attributable to the risk factors (see online supplementary material, Supplemental Table 1).

To determine the mortality attributable to risk factors, first, the population attributable fraction (PAF) was determined using the following inputs: the exposure level for each dietary risk factor $(P)$; the effect size (RR); the TMREL; and the total number of deaths from the specific disease. Joint PAF were calculated for wasting, underweight and stunting assuming independency. Additional details on methods of estimation can be found elsewhere ${ }^{(2)}$.

The PAF was given as ${ }^{(2)}$

$$
P A F_{\text {joasgt }}=\frac{\int_{x=l}^{u} R R_{\text {joast }}(x) P_{\text {jasgt }}(x) \mathrm{d} x-R R_{\text {joasg }}\left(T M R E L_{\text {jas }}\right)}{\int_{x=l}^{u} R R_{\text {joasg }}(x) P_{\text {jasgt }}(x) \mathrm{d} x}
$$

for continuous risk factors, or as

$$
P A F_{\text {joasgt }}=\frac{\sum_{x=l}^{u} R R_{\text {joast }}(x) P_{\text {jasgt }}(x)-R R_{\text {joasg }}\left(T M R E L_{\text {jas }}\right)}{\sum_{x=l}^{u} R R_{\text {joas }}(x) P_{\text {jasgt }}(x)}
$$

for dichotomous or polychotomous risk factors, where:

$P A F_{\text {joasgt }}$ is a population attributable fraction for a risk factor $j$, attributed to cause $o$ for age group $a$, sex $s$, geography $g$ and year $t$;

$R R_{\text {joasg }}(x)$ is the relative risk as a function of exposure level $x$ for a risk factor $j$, attributed to cause $o$, age group $a$, sex $s$ and geography $g$, with the lowest level of observed exposure being $l$ and the highest being $u$;

$P_{\text {jasgt }}(x)$ is the distribution of exposure at $x$ for age group $a$, sex $s$, geography $g$ and year $t$; and

$T M R E L_{j a s}$ is the TMREL for risk factor $j$, age group $a$ and $\operatorname{sex} s$. 
Then, the total attributable mortality was determined using the number of deaths and $\mathrm{PAF}^{(2)}$, with the GBD 2015 mortality study used to obtain the number of deaths ${ }^{(2,21)}$ : Total attributable burden $_{\text {jasgt }}=\sum_{o=1}^{w}$ Burden $_{\text {joasgt }} P A F_{\text {joasgt }}$

The overall proportion of disease burden attributable to aggregated dietary risk factors and CMU was computed using the following formula ${ }^{(2)}$ :

$$
P A F_{\text {joasgt }}=1-\prod_{k=1}^{K}\left[1-P A F_{\text {joasgt }} \prod_{k=1}^{K}\left(1-M F_{j i o}\right)\right]
$$

where:

$K$ is a set of risk factors for the aggregation;

$P A F_{\text {joasgt }}$ is PAF for risk $j$ for age group $a$, sex $s$, geography $g$ and year $t$; and

$M F_{j i o}$ is the mediation factor for risk $j$ mediated through $i$ for cause $o$.

Using the Monte Carlo approach ${ }^{(29)}$, the uncertainty of parameters for exposure, relative risk and attributable mortality was calculated with 1000 repeated draws. Uncertainty intervals (95\% UI) for the estimates were reported ${ }^{(2)}$.

\section{Results}

\section{Mortality related to child and maternal undernutrition, dietary risk factors and overweight/obesity in 2015}

Table 1 provides the estimated number, crude rates and proportions of mortality accounted for by CMU, diet and overweight/obesity in SSA and its sub-regions in 2015. Deaths attributable to CMU totalled 826204 (95\% UI $737346,923789)$ or $10 \cdot 3 \%$ (95\% UI 9.1, $11 \cdot 6 \%$ ) of all deaths in SSA. The proportion of mortality caused by CMNN diseases attributable to CMU was $17 \cdot 7 \%$ (95\% UI $15 \cdot 7,19.9 \%)$. Except for Southern SSA where dietary factors of NCD contributed the highest number of deaths, in all other sub-regions CMU was responsible for the highest number of deaths. Overweight/obesity and dietary risk factors accounted for 266768 (95\% UI 189051,353096 ) or $3.3 \%$ (95\% UI 2.4, 4.4\%) and 558578 (95\% UI 453433 , 680197 ) or $7 \cdot 0 \%$ (95\% UI 5.8, 8.3\%) of all-cause mortality, respectively. The relative contribution to NCD mortality attributable to overweight/obesity and dietary risk factors was $9 \cdot 8 \%$ (95\% UI $7 \cdot 0,12 \cdot 8 \%$ ) and $20 \cdot 5 \%$ (95\% UI $17 \cdot 3$, $24.3 \%)$, respectively. The age-standardized proportion of all-cause mortality attributable to CMU was higher in Western SSA compared with other sub-regions.

In 2015, the age-standardized proportion of all-cause mortality was significantly higher for dietary risks of NCD compared with overweight/obesity and CMU. Overall, the age-standardized proportion of deaths attributable to
CMU, overweight/obesity and dietary risks was $12 \cdot 2 \%$ (95\% UI 10.2, 14.7\%), 3.8\% (95\% UI 3.4, $4 \cdot 3 \%$ ) and $5 \cdot 3 \%$ (95\% UI 3.8, 7.0\%), respectively (Table 2 ). The highest age-standardized proportion of deaths attributable to CMU was found in Chad $(7 \cdot 8 \% ; 95 \%$ UI $4.8,11 \cdot 8 \%)$. On the other hand, the highest age-standardized proportion of deaths attributable to overweight/obesity and dietary risks of NCD was found in South Africa (11.6\%; $95 \%$ UI 9.4, $13.7 \%)$ and Cameroon (17.8\%; 95\% UI 15.0, 21.2\%), respectively. Botswana (0.7\%; $95 \%$ UI 0.3, 1.4\%), Burundi (1.5\%; $95 \%$ UI 0.6, 2.7\%) and Guinea-Bissau (6.7\%; $95 \%$ UI $5 \cdot 6,7 \cdot 9 \%$ ) had the lowest age-standardized proportion of mortality attributable to CMU, overweight/obesity and dietary risks of NCD, respectively (see online supplementary material, Supplemental Tables 6, 7 and 8).

The number, rate and proportion of deaths attributable to CMU, overweight/obesity and dietary risks of NCD by age are shown in Fig. 2 and the online supplementary material, Supplemental Fig. 1.

Childhood wasting and underweight were the most common CMU risks, accounting for 8.5 and $3.0 \%$ of all deaths, respectively. Diets low in fruits $(2 \cdot 2 \%)$, whole grains $(1.7 \%)$, vegetables (1.5\%), nuts and seeds (1.3\%) and high in $\mathrm{Na}(1.3 \%)$ were found to be the most common dietary risk factors (Table 3 ).

\section{Trend of mortality attributable to child and maternal undernutrition, dietary risk factors and overweight/obesity between 1990 and 2015}

Table 3 depicts the trends of mortality attributable to CMU, overweight/obesity and dietary risks of NCD in SSA and sub-regions. The number of deaths attributable to CMU decreased substantially by $44.6 \%$ in the past 25 years, falling from 1.49 million (1490671; 95\% UI 1305548 , 1667532 ) in 1990 to 0.83 million (826 204; $95 \%$ UI 737346 , $923789)$ in 2015. In contrast, deaths attributable to overweight/obesity and dietary risks of NCD increased by 143.8 and $73.0 \%$, rising from 109407 (95\% UI 71 767, 152893) in 1990 to 266768 (95\% UI 189051,353096 ) in 2015 and from 322932 (95\% UI 271234, 387047 ) to 558578 (95\% UI 453433 , 680 197), respectively. Similar patterns were identified at sub-regional level. The age-standardized proportion of deaths related to by CMU decreased by $55 \cdot 3 \%$, falling from $8.5 \%$ (95\% UI 7.4, 9.5\%) in 1990 to $3.8 \%$ (95\% UI $3 \cdot 4,4 \cdot 3 \%)$ in 2015 . On the other hand, the agestandardized proportion of deaths attributable to overweight/obesity and dietary risks of NCD rose by 63.3 and $17 \cdot 2 \%$, rising from $3 \cdot 2 \%$ (95\% UI $2 \cdot 1,4 \cdot 6 \%$ ) and $10 \cdot 4 \%$ (95\% UI $8 \cdot 7,12.6 \%$ ) in 1990 to $5.3 \%$ (95\% UI 3.8, $7 \cdot 0 \%$ ) and $12.2 \%$ (95\% UI 10.2, $14.7 \%$ ) in 2015 , respectively. However, in the Southern SSA sub-region, the proportion of deaths attributable to overweight/obesity decreased by $10 \cdot 3 \%$, falling from $14.9 \%$ (95\% UI $12 \cdot 6,17 \cdot 9 \%$ ) to $13.4 \%$ (95\% UI 11.3, 15.9\%). The attributable mortality trend across the past 25 years is shown in Fig. 3 and the online supplementary material, Supplemental Fig. 2. 
Table 1 The mortality associated with child and maternal undernutrition (CMU), overweight/obesity and dietary risks of non-communicable diseases (NCD) in sub-Saharan Africa by sex and sub-region, 2015

\begin{tabular}{|c|c|c|c|c|c|c|c|c|c|}
\hline Sub-region/risk factor & Sex & Number & $95 \%$ UI & $\begin{array}{c}\text { Crude rate } \\
\text { (per 100 000) }\end{array}$ & $95 \%$ UI & $\begin{array}{c}\text { Crude proportion (\%), } \\
\text { all-cause }\end{array}$ & $95 \%$ UI & $\begin{array}{c}\text { Crude proportion (\%), } \\
\text { CMNND or NCD } \neq\end{array}$ & $95 \%$ UI \\
\hline \multicolumn{10}{|c|}{ Central sub-Saharan Africa } \\
\hline \multirow{3}{*}{ CMU } & Males & 60921 & 44674,85600 & 107 & 78,150 & 11.5 & $8 \cdot 0,16 \cdot 7$ & $20 \cdot 3$ & $15 \cdot 0,27 \cdot 4$ \\
\hline & Females & 51993 & 38745,67891 & 90 & 67,118 & $10 \cdot 3$ & $7 \cdot 5,13 \cdot 8$ & $17 \cdot 6$ & $13 \cdot 4,22 \cdot 2$ \\
\hline & Both sexes & 112914 & 86048,147814 & 98 & 75,129 & $10 \cdot 8$ & $8 \cdot 3,14 \cdot 4$ & $18 \cdot 9$ & $15 \cdot 1,23 \cdot 8$ \\
\hline \multirow[t]{3}{*}{ Dietary risks of NCD } & Males & 34928 & 20768,53734 & 61 & 36,94 & $6 \cdot 4$ & $4.5,8 \cdot 6$ & $19 \cdot 6$ & $15 \cdot 3,24 \cdot 0$ \\
\hline & Females & 38562 & 22618,59551 & 67 & 39,103 & 7.4 & $5 \cdot 2,9 \cdot 8$ & $20 \cdot 4$ & $16 \cdot 1,25 \cdot 4$ \\
\hline & Both sexes & 73490 & 45379,107237 & 64 & 40,93 & $7 \cdot 0$ & $4 \cdot 9,9 \cdot 0$ & $20 \cdot 0$ & $16 \cdot 0,24 \cdot 7$ \\
\hline \multirow[t]{3}{*}{ Overweight/obesity } & Males & 11601 & 4642, 22671 & 20 & 8,40 & $2 \cdot 1$ & $1 \cdot 0,3 \cdot 8$ & $6 \cdot 5$ & $3 \cdot 2,10 \cdot 7$ \\
\hline & Females & 17412 & 7962,32278 & 30 & 14,56 & $3 \cdot 3$ & $1 \cdot 9,5 \cdot 3$ & $9 \cdot 2$ & $5 \cdot 3,13.9$ \\
\hline & Both sexes & 29013 & 15050,51043 & 25 & 13,44 & $2 \cdot 7$ & $1 \cdot 6,4 \cdot 2$ & 7.9 & $4.9,11.4$ \\
\hline \multicolumn{10}{|c|}{ Eastern sub-Saharan Africa } \\
\hline \multirow[t]{3}{*}{ CMU } & Males & 149789 & 131170,171630 & 80 & 70,92 & $9 \cdot 6$ & $8 \cdot 0,11.5$ & $17 \cdot 6$ & $15 \cdot 3,20 \cdot 3$ \\
\hline & Females & 133530 & 114345,153324 & 70 & 60,81 & $9 \cdot 7$ & $8 \cdot 0,11 \cdot 6$ & $17 \cdot 1$ & $14 \cdot 6,19 \cdot 6$ \\
\hline & Both sexes & 283319 & 250895,320673 & 75 & 67,85 & $9 \cdot 6$ & $8 \cdot 3,11 \cdot 2$ & $17 \cdot 4$ & $15 \cdot 3,19 \cdot 7$ \\
\hline \multirow[t]{3}{*}{ Dietary risks of NCD } & Males & 119901 & 87703,164985 & 64 & 47,88 & $7 \cdot 6$ & $6 \cdot 2,9 \cdot 5$ & $21 \cdot 6$ & $18 \cdot 0,25 \cdot 7$ \\
\hline & Females & 102730 & 70418,146151 & 54 & 37,77 & 7.4 & $5 \cdot 7,9 \cdot 6$ & $19 \cdot 7$ & $15 \cdot 8,24 \cdot 4$ \\
\hline & Both sexes & 222631 & 171455,293795 & 59 & 45,78 & 7.5 & $6 \cdot 1,9 \cdot 2$ & $20 \cdot 7$ & $17 \cdot 1,25 \cdot 0$ \\
\hline \multirow[t]{3}{*}{ Overweight/obesity } & Males & 30374 & 14290,54154 & 16 & 8,29 & 1.9 & $0.9,3 \cdot 2$ & 5.5 & $2 \cdot 7,8 \cdot 9$ \\
\hline & Females & 46043 & 25932,72884 & 24 & 14,38 & $3 \cdot 3$ & $2.0,4.9$ & $8 \cdot 8$ & $5 \cdot 4,12 \cdot 7$ \\
\hline & Both sexes & 76417 & 46080,111311 & 20 & 12,30 & $2 \cdot 6$ & $1 \cdot 6,3 \cdot 7$ & $7 \cdot 1$ & $4 \cdot 6,9.9$ \\
\hline \multicolumn{10}{|c|}{ Southern sub-Saharan Africa } \\
\hline \multirow[t]{3}{*}{$\mathrm{CMU}$} & Males & 10373 & 8848,12082 & 27 & 23,32 & $2 \cdot 4$ & $2 \cdot 0,2 \cdot 9$ & $5 \cdot 1$ & $4 \cdot 3,5 \cdot 9$ \\
\hline & Females & 9090 & 7750,10561 & 23 & 20,27 & $2 \cdot 3$ & $1 \cdot 9,2 \cdot 7$ & 4.8 & $4 \cdot 0,5 \cdot 6$ \\
\hline & Both sexes & 19463 & 16718,22467 & 25 & 22,29 & $2 \cdot 4$ & $2 \cdot 0,2 \cdot 7$ & 4.9 & $4 \cdot 2,5 \cdot 7$ \\
\hline \multirow[t]{3}{*}{ Dietary risks of NCD } & Males & 39752 & 32162,48409 & 104 & 84,127 & $9 \cdot 3$ & $7 \cdot 9,11$ & $24 \cdot 2$ & $20 \cdot 7,28 \cdot 3$ \\
\hline & Females & 45292 & 36735,55600 & 115 & 93,141 & 11.4 & $9 \cdot 6,13 \cdot 7$ & $24 \cdot 3$ & $20 \cdot 7,28 \cdot 8$ \\
\hline & Both sexes & 85044 & 70412,102766 & 110 & 91,133 & $10 \cdot 3$ & $8 \cdot 7,12 \cdot 2$ & $24 \cdot 3$ & $20 \cdot 8,28 \cdot 5$ \\
\hline \multirow[t]{3}{*}{ Overweight/obesity } & Males & 23800 & 15959,32345 & 62 & 42,85 & 5.6 & $3 \cdot 8,7.4$ & $14 \cdot 5$ & $10 \cdot 0,19 \cdot 2$ \\
\hline & Females & 44089 & 34540,54775 & 112 & 88,139 & $11 \cdot 1$ & $9 \cdot 0,13 \cdot 3$ & 23.7 & $19 \cdot 5,27 \cdot 9$ \\
\hline & Both sexes & 67889 & 54186,81717 & 88 & 70,106 & $8 \cdot 2$ & $6 \cdot 7,9 \cdot 8$ & $19 \cdot 4$ & $15 \cdot 8,23 \cdot 0$ \\
\hline \multicolumn{10}{|c|}{ Western sub-Saharan Africa } \\
\hline \multirow[t]{3}{*}{$\mathrm{CMU}$} & Males & 218342 & 181853,259496 & 111 & 92,132 & $12 \cdot 8$ & $10 \cdot 3,15 \cdot 2$ & $20 \cdot 2$ & $16 \cdot 6,23 \cdot 7$ \\
\hline & Females & 192166 & 160756,229348 & 99 & 83,118 & $13 \cdot 1$ & $10 \cdot 7,15 \cdot 8$ & $20 \cdot 0$ & $16 \cdot 8,23 \cdot 7$ \\
\hline & Both sexes & 410508 & 348886,476136 & 105 & 89,122 & $12 \cdot 9$ & $10 \cdot 8,15 \cdot 1$ & $20 \cdot 1$ & $17 \cdot 0,23 \cdot 3$ \\
\hline \multirow{3}{*}{ Dietary risks of NCD } & Males & 89085 & 69374,116474 & 45 & 35,59 & $5 \cdot 2$ & $4 \cdot 3,6 \cdot 3$ & $18 \cdot 2$ & $15 \cdot 3,21 \cdot 7$ \\
\hline & Females & 88328 & 66490,121954 & 46 & 34,63 & $6 \cdot 0$ & $4.9,7.5$ & $19 \cdot 7$ & $16 \cdot 0,23 \cdot 8$ \\
\hline & Both sexes & 177413 & 140988,225225 & 45 & 36,58 & $5 \cdot 6$ & $4 \cdot 6,6 \cdot 7$ & $18 \cdot 9$ & $16 \cdot 0,22 \cdot 4$ \\
\hline \multirow[t]{3}{*}{ Overweight/obesity } & Males & 38131 & 20308,61072 & 19 & 10,31 & $2 \cdot 2$ & $1.2,3.4$ & $7 \cdot 8$ & $4.5,11 \cdot 7$ \\
\hline & Females & 55319 & 33901,82816 & 29 & 18,43 & 3.7 & $2 \cdot 5,5 \cdot 1$ & $12 \cdot 3$ & $8 \cdot 4,16 \cdot 4$ \\
\hline & Both sexes & 93450 & 63598,130648 & 24 & 16,33 & $2 \cdot 9$ & $2 \cdot 1,3.9$ & $10 \cdot 0$ & $7 \cdot 0,13 \cdot 1$ \\
\hline \multicolumn{10}{|l|}{ Sub-Saharan Africa } \\
\hline \multirow{3}{*}{$\mathrm{CMU}$} & Males & 439426 & 385540,496385 & 92 & 80,103 & $10 \cdot 4$ & $9 \cdot 0,11 \cdot 8$ & $18 \cdot 0$ & $15 \cdot 7,20 \cdot 4$ \\
\hline & Females & 386778 & 343498,438507 & 81 & 72,91 & $10 \cdot 3$ & $9 \cdot 0,11 \cdot 8$ & $17 \cdot 4$ & $15 \cdot 3,19 \cdot 8$ \\
\hline & Both sexes & 826204 & 737346,923789 & 86 & 77,96 & $10 \cdot 3$ & $9 \cdot 1,11 \cdot 6$ & $17 \cdot 7$ & $15 \cdot 7,19 \cdot 9$ \\
\hline \multirow[t]{3}{*}{ Dietary risks of NCD } & Males & 283666 & 228502,351697 & 59 & 48,73 & $6 \cdot 7$ & $5 \cdot 6,8 \cdot 0$ & $20 \cdot 5$ & $17 \cdot 3,24 \cdot 2$ \\
\hline & Females & 274912 & 215903,352108 & 57 & 45,73 & $7 \cdot 3$ & $6.0,8.9$ & $20 \cdot 4$ & $17 \cdot 2,24 \cdot 7$ \\
\hline & Both sexes & 558578 & 453433,680197 & 58 & 47,71 & $7 \cdot 0$ & $5 \cdot 8,8 \cdot 3$ & 20.5 & $17 \cdot 3,24 \cdot 3$ \\
\hline Overweight/obesity & Males & 103906 & 58575,158084 & 22 & 12,33 & $2 \cdot 4$ & $1.4,3.6$ & 7.5 & $4.4,11 \cdot 1$ \\
\hline & Females & 162862 & 111690,219247 & 34 & 23,46 & $4 \cdot 3$ & $3 \cdot 0,5 \cdot 7$ & $12 \cdot 1$ & $8 \cdot 5,15 \cdot 9$ \\
\hline & Both sexes & 266768 & 189051,353096 & 28 & 20,37 & 3.3 & $2 \cdot 4,4.4$ & $9 \cdot 8$ & $7 \cdot 0,12 \cdot 8$ \\
\hline
\end{tabular}

UI, uncertainty interval; CMNND, communicable, maternal, neonatal and nutritional diseases.

tProportions are for all causes of death.

†Proportions are for specific categories of diseases (i.e. CMNND accounting for CMU; NCD accounting for dietary risks and overweight/obesity). 
Table 2 The burden of mortality associated with child and maternal undernutrition (CMU), overweight/obesity and dietary risks of non-communicable diseases (NCD) in sub-Saharan Africa between 1990 and 2015

\begin{tabular}{|c|c|c|c|c|c|c|c|c|c|c|c|c|c|c|c|c|c|c|c|c|c|c|}
\hline \multirow[b]{2}{*}{$\begin{array}{l}\text { Sub-region/Risk } \\
\text { factor }\end{array}$} & \multicolumn{6}{|c|}{ Number } & \multicolumn{4}{|c|}{$\begin{array}{l}\text { Age-standardized rate } \\
\text { (per } 100000)\end{array}$} & \multicolumn{6}{|c|}{$\begin{array}{l}\text { Age-standardized proportion (\%), } \\
\text { all-causef }\end{array}$} & \multicolumn{6}{|c|}{$\begin{array}{l}\text { Age-standardized proportion (\%), } \\
\text { CMNND or NCD } \neq\end{array}$} \\
\hline & 1990 & $95 \%$ UI & 2015 & $95 \%$ UI & $\begin{array}{c}\text { Change } \\
(\%)\end{array}$ & $95 \%$ UI & 1990 & $95 \%$ UI & 2015 & $95 \%$ UI & 1990 & $95 \%$ UI & 2015 & $95 \%$ UI & $\begin{array}{c}\text { Change } \\
(\%)\end{array}$ & $95 \%$ UI & 1990 & $95 \% \mathrm{UI}$ & 2015 & $95 \%$ UI & $\begin{array}{c}\text { Change } \\
(\%)\end{array}$ & $95 \%$ UI \\
\hline \multicolumn{23}{|c|}{ Central sub-Saharan Africa } \\
\hline $\begin{array}{l}\text { CMU } \\
\text { Dietary risks of }\end{array}$ & $\begin{array}{r}152378 \\
37715\end{array}$ & $\begin{array}{c}123661,190511 \\
25752,53853\end{array}$ & $\begin{array}{r}112914 \\
73490\end{array}$ & $\begin{array}{l}86048,147814 \\
45379,107237\end{array}$ & $\begin{array}{r}-26 \\
95\end{array}$ & $\begin{aligned}-45,-1 \\
25,203\end{aligned}$ & $\begin{array}{l}144 \\
206\end{array}$ & $\begin{array}{l}118,179 \\
141,292\end{array}$ & $\begin{array}{r}60 \\
194\end{array}$ & $\begin{array}{c}45,80 \\
121,276\end{array}$ & $\begin{array}{r}7.5 \\
10.7\end{array}$ & $\begin{array}{l}5 \cdot 9,9 \cdot 8 \\
8 \cdot 1,13 \cdot 6\end{array}$ & $\begin{array}{c}3 \cdot 9^{*} \\
12 \cdot 4\end{array}$ & $\begin{array}{l}2 \cdot 9,5 \cdot 6 \\
9 \cdot 1,15 \cdot 7\end{array}$ & $\begin{array}{r}-48.4 \\
16.0\end{array}$ & $\begin{array}{c}-63 \cdot 5,-28 \cdot 3 \\
3 \cdot 6,27 \cdot 8\end{array}$ & $\begin{array}{l}15 \cdot 9 \\
22 \cdot 9\end{array}$ & $\begin{array}{l}12 \cdot 8,19 \cdot 6 \\
18 \cdot 8,27 \cdot 9\end{array}$ & $\begin{array}{ll}6 & 9.8^{*} \\
9 & 22.7\end{array}$ & $\begin{array}{r}7.2,12 \cdot 8 \\
18 \cdot 5,28 \cdot 1\end{array}$ & $\begin{array}{r}-38.6 \\
-0.8\end{array}$ & $\begin{array}{l}-55 \cdot 5,-17 \cdot 6 \\
-6 \cdot 4,4 \cdot 6\end{array}$ \\
\hline $\begin{array}{l}\mathrm{NCD} \\
\text { Overweight/ } \\
\text { obesity }\end{array}$ & 13683 & 7705,21628 & 29013 & 15050,51043 & 112 & 30,259 & 67 & 38,105 & 68 & 37,116 & 3.5 & $2 \cdot 1,5 \cdot 0$ & $4 \cdot 3$ & $2 \cdot 6,6 \cdot 5$ & 24.7 & $1 \cdot 0,63 \cdot 8$ & 7.5 & $4.5,10 \cdot 8$ & 88.0 & $5 \cdot 0,11 \cdot 6$ & 6.7 & $-11 \cdot 9,36 \cdot 7$ \\
\hline \multicolumn{23}{|c|}{$\begin{array}{l}\text { obesity } \\
\text { Eastern sub-Saharan Africa }\end{array}$} \\
\hline CMU & $\begin{array}{l}605387 \\
135083\end{array}$ & $\begin{array}{l}529428,684327 \\
111972,166003\end{array}$ & $\begin{array}{l}283319 \\
222631\end{array}$ & $\begin{array}{l}250895,320673 \\
171455,293795\end{array}$ & $\begin{array}{r}-53 \\
65\end{array}$ & $\begin{array}{r}-60,-45 \\
31,103\end{array}$ & $\begin{array}{l}169 \\
213\end{array}$ & $\begin{array}{l}149,190 \\
176,265\end{array}$ & $\begin{array}{r}52 \\
173\end{array}$ & $\begin{array}{c}46,59 \\
132,226\end{array}$ & $\begin{array}{r}8.1 \\
10 \cdot 2\end{array}$ & $\begin{array}{l}7 \cdot 1,9 \cdot 2 \\
8 \cdot 5,12 \cdot 5\end{array}$ & $12 \cdot 4^{*}$ & $\begin{array}{l}3 \cdot 2,4 \cdot 3 \\
10 \cdot 2,15 \cdot 1\end{array}$ & $\begin{array}{r}-54.1 \\
21.2\end{array}$ & $\begin{array}{c}-61 \cdot 3,-45 \cdot 6 \\
10 \cdot 8,31 \cdot 2\end{array}$ & $\begin{array}{l}16 \cdot 7 \\
23 \cdot 6\end{array}$ & $\begin{array}{l}14 \cdot 6,19 \cdot 0 \\
19 \cdot 7,28 \cdot 7\end{array}$ & $\begin{array}{ll}0 & 9 \cdot 3^{*} \\
7 & 23 \cdot 5\end{array}$ & $\begin{array}{r}8 \cdot 2,10 \cdot 6 \\
19 \cdot 4,28 \cdot 7\end{array}$ & $\begin{array}{r}-44 \cdot 7 \\
-0.4\end{array}$ & $\begin{array}{l}-52 \cdot 9,-35 \cdot 3 \\
-6 \cdot 7,5.5\end{array}$ \\
\hline $\begin{array}{l}\text { NCD } \\
\text { Overweight/ } \\
\text { obesity }\end{array}$ & 32585 & 18538,49585 & 76417 & 46080,111311 & 135 & 83,201 & 46 & 26,72 & 54 & 33,80 & $2 \cdot 2$ & $1 \cdot 2,3 \cdot 4$ & 3.9 & $2 \cdot 4,5.5$ & 74.9 & $50 \cdot 2,107 \cdot 6$ & 5.1 & $2 \cdot 9,7 \cdot 9$ & 7.4 & $4.7,10.5$ & $43 \cdot 9$ & $25 \cdot 3,70 \cdot 2$ \\
\hline \multicolumn{23}{|c|}{ Southern sub-Saharan Africa } \\
\hline $\begin{array}{l}\mathrm{CMU} \\
\text { Dietary risks of }\end{array}$ & $\begin{array}{l}52501 \\
50079\end{array}$ & $\begin{array}{l}49300,55991 \\
42299,59924\end{array}$ & $\begin{array}{l}19463 \\
85044\end{array}$ & $\begin{array}{l}16718,22467 \\
70412,102766\end{array}$ & $\begin{array}{r}-63 \\
70\end{array}$ & $\begin{array}{l}-68,-57 \\
51,94\end{array}$ & $\begin{array}{r}61 \\
219\end{array}$ & $\begin{array}{c}58,65 \\
184,263\end{array}$ & $\begin{array}{r}20 \\
204\end{array}$ & $\begin{array}{c}17,23 \\
169,246\end{array}$ & $\begin{array}{r}4.2 \\
14.9\end{array}$ & $\begin{array}{l}3 \cdot 9,4 \cdot 5 \\
12 \cdot 6,17 \cdot 9\end{array}$ & $\begin{array}{c}1 \cdot 3^{\star} \\
13 \cdot 4\end{array}$ & $\begin{array}{l}1 \cdot 1,1 \cdot 5 \\
11 \cdot 3,15 \cdot 9\end{array}$ & $\begin{array}{l}-68 \cdot 2 \\
-10 \cdot 3\end{array}$ & $\begin{array}{l}-73 \cdot 0,-63 \cdot 1 \\
-14 \cdot 9,-4 \cdot 9\end{array}$ & $\begin{array}{l}14 \cdot 9 \\
24 \cdot 2\end{array}$ & $\begin{array}{l}13 \cdot 4,16 \cdot 2 \\
20 \cdot 6,28 \cdot 7\end{array}$ & $\begin{array}{l}2 \\
7.5^{*} \\
24.8\end{array}$ & $\begin{array}{c}3 \cdot 0,4 \cdot 0 \\
21 \cdot 1,29 \cdot 3\end{array}$ & $\begin{array}{r}-76.8 \\
2.4\end{array}$ & $\begin{array}{l}-80 \cdot 5,-72 \cdot 8 \\
-0.2,6 \cdot 1\end{array}$ \\
\hline $\begin{array}{c}\text { Overweight/ } \\
\text { obesity }\end{array}$ & 35031 & 27735,42711 & 67889 & 54186,81717 & 94 & 68,124 & 146 & 114,180 & 157 & 124,190 & 9.9 & $7 \cdot 8,12 \cdot 1$ & $10 \cdot 4$ & $8 \cdot 3,12 \cdot 4$ & 4.2 & $-4 \cdot 4,13 \cdot 5$ & $16 \cdot 1$ & $12 \cdot 7,19 \cdot 7$ & 719.2 & $15 \cdot 5,23 \cdot 0$ & 19.0 & $10 \cdot 9,27 \cdot 2$ \\
\hline \multicolumn{23}{|c|}{$\begin{array}{l}\text { Westeressity Saharan Africa } \\
\text { CMU } \\
680406 \quad 577609\end{array}$} \\
\hline $\begin{array}{l}\text { CMU } \\
\text { Dietary risks of } \\
\text { NCD }\end{array}$ & $\begin{array}{l}680406 \\
100056\end{array}$ & $\begin{array}{r}577609,777762 \\
78235,127200\end{array}$ & $\begin{array}{l}410508 \\
177413\end{array}$ & $\begin{array}{l}348886,476136 \\
140988,225225\end{array}$ & $\begin{array}{r}-40 \\
77\end{array}$ & $\begin{array}{r}-49,-28 \\
39,128\end{array}$ & $\begin{array}{l}170 \\
151\end{array}$ & $\begin{array}{l}145,195 \\
119,191\end{array}$ & $\begin{array}{r}57 \\
144\end{array}$ & $\begin{array}{c}49,66 \\
115,183\end{array}$ & $\begin{array}{r}10.3 \\
9.2\end{array}$ & $\begin{array}{l}8.5,12 \cdot 2 \\
7 \cdot 6,11 \cdot 0\end{array}$ & $\begin{array}{c}415^{x} \\
11 \cdot 4\end{array}$ & $\begin{array}{l}3 \cdot 7,5 \cdot 4 \\
9 \cdot 6,13 \cdot 7\end{array}$ & $\begin{array}{r}-56.2 \\
24.9\end{array}$ & $\begin{array}{c}-65 \cdot 2,-45 \cdot 2 \\
16 \cdot 8,33 \cdot 9\end{array}$ & $\begin{array}{l}20 \cdot 3 \\
21 \cdot 2\end{array}$ & $\begin{array}{l}17.0,23 \cdot 6 \\
17 \cdot 9,25 \cdot 2\end{array}$ & $\begin{array}{ll}0 & 10.3 \\
2 & 23.0\end{array}$ & $\begin{array}{r}8 \cdot 4,12 \cdot 2 \\
19 \cdot 4,27 \cdot 2\end{array}$ & $\begin{array}{r}-49.4 \\
8.4\end{array}$ & $\begin{array}{c}-58.5,-3.9 \\
4.5,12.5\end{array}$ \\
\hline $\begin{array}{l}\text { Overweight/ } \\
\text { obesity } \\
\text { Sub-Saharan Afric }\end{array}$ & 28107 & 15240,44439 & 93450 & 63598,130648 & 232 & 136,390 & 34 & 18,55 & 64 & 42,89 & $2 \cdot 1$ & $1 \cdot 1,3 \cdot 3$ & $5 \cdot 0^{*}$ & $3.5,6.7$ & 141.1 & $92 \cdot 5,228 \cdot 4$ & 4.8 & $2 \cdot 6,7.5$ & $10 \cdot 1$ & $7 \cdot 0,13 \cdot 5$ & 109.2 & $70 \cdot 2,180$ \\
\hline CMU & 1490671 & 1305548,1667532 & 826204 & 737346,923789 & -45 & $-51,-37$ & 156 & 138,174 & 53 & 47,59 & 8.5 & $7.4,9.5$ & $3 \cdot 8^{*}$ & & $-55 \cdot 2$ & $-61 \cdot 1,-49 \cdot 2$ & $18 \cdot 2$ & $15 \cdot 9,20 \cdot 3$ & $\begin{array}{ll}3 & 9 \cdot 3^{*}\end{array}$ & $8 \cdot 2,10 \cdot 6$ & -48.8 & $-55 \cdot 2,-41 \cdot 8$ \\
\hline $\begin{array}{l}\text { Dietary risks of } \\
\text { NCD }\end{array}$ & 322932 & & 558578 & 453433,680197 & 73 & 52,97 & 191 & 158,231 & 169 & 138,208 & 10.4 & $8 \cdot 7,12 \cdot 6$ & $12 \cdot 2$ & $10 \cdot 2,14 \cdot 7$ & $17 \cdot 2$ & $11 \cdot 9,22 \cdot 5$ & $22 \cdot 8$ & $19 \cdot 2,27 \cdot 2$ & $223 \cdot 3$ & $19 \cdot 6,28 \cdot 0$ & 2.5 & $-1 \cdot 0,5 \cdot 6$ \\
\hline $\begin{array}{l}\text { Overweight/ } \\
\text { obesity }\end{array}$ & 109407 & 71767,152893 & 266768 & 189051,353096 & 144 & 108,195 & 59 & 39,83 & 73 & 51,97 & 3.2 & $2 \cdot 1,4 \cdot 6$ & 5.3 & $3 \cdot 8,7 \cdot 0$ & $63 \cdot 3$ & $47 \cdot 9,83.5$ & 7.1 & $4 \cdot 6,10 \cdot 0$ & $010 \cdot 1$ & $7 \cdot 2,13 \cdot 4$ & $42 \cdot 9$ & $30 \cdot 3,60 \cdot 1$ \\
\hline
\end{tabular}

UI, uncertainty interval; CMNND, communicable, maternal, neonatal and nutritional diseases.

* Significantly increased or decreased based on $95 \% \mathrm{UI}$.

†Proportions are for specific category of diseases (i.e. CMNND accounting for CMU; NCD accounting for dietary risks and high- overweight/obesity). 

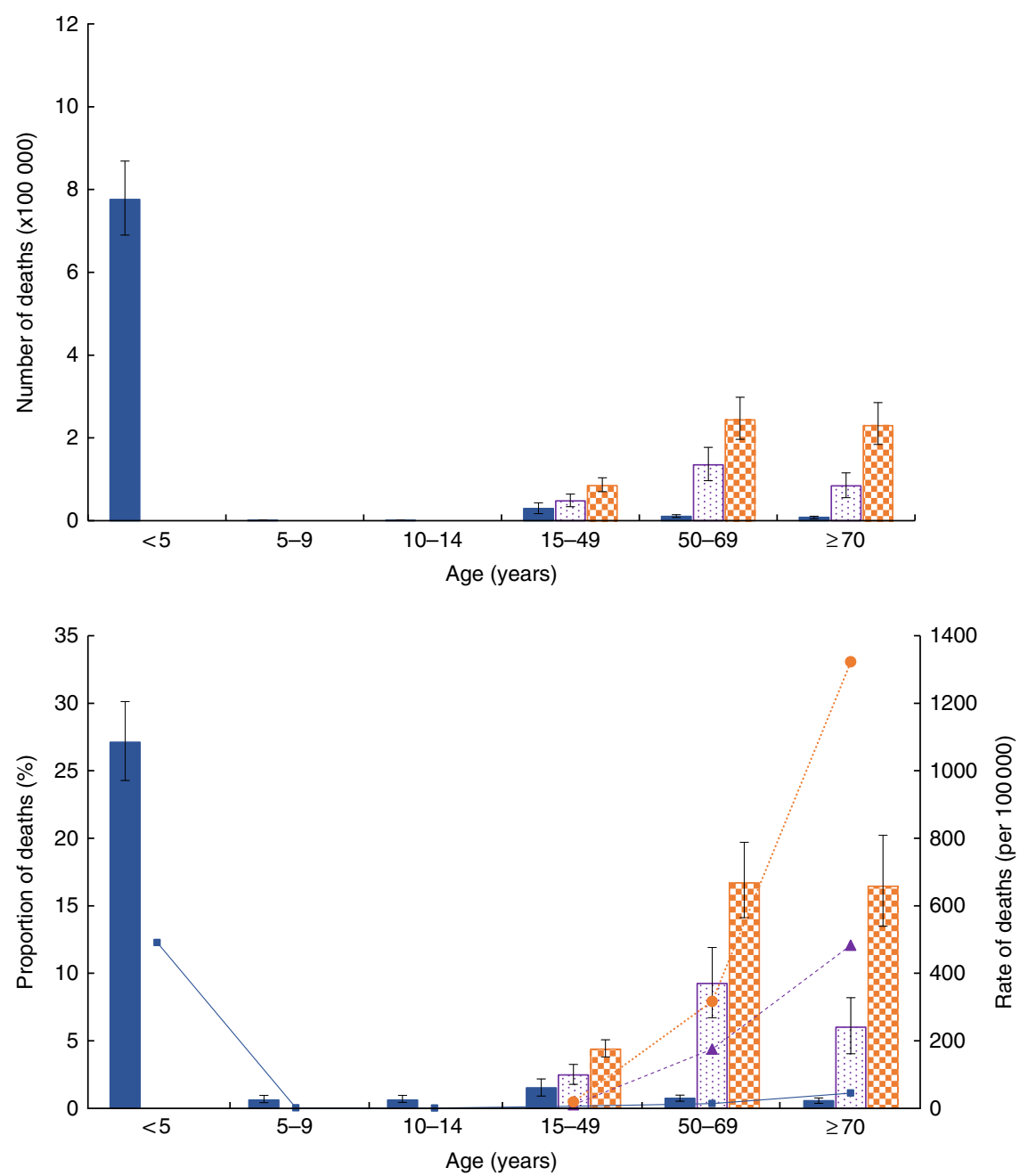

Fig. 2 (colour online) Number, proportion (of all-cause) and rate of deaths associated with child and maternal undernutrition (CMU), overweight/obesity and dietary risks of non-communicable diseases (NCD) in sub-Saharan Africa by age in 2015: $\square$, CMU number/proportion; $\rightarrow-$, CMU rate; $:$, overweight/obesity number/proportion; -...-., overweight/obesity rate; 8 , dietary risks of NCD number/proportion; ........., dietary risks of NCD rate. Numbers and proportions are mean values with their $95 \%$ uncertainty intervals represented by vertical bars

Countries and sub-regions show different levels of increase or decrease in mortality attributable to each of the risk factors. The age-standardized proportion of deaths attributable to CMU fell by more than half in most of the countries. The highest reduction ( $80 \%$ or above) was found in Botswana, South Africa and Cape Verde. The age-standardized proportion of deaths attributable to overweight/obesity rose in all SSA sub-regions. The highest increase (140.7\%) was recorded in Western SSA, rising from $2 \cdot 1 \%$ (95\% UI $1 \cdot 1,3 \cdot 3 \%$ ) in 1990 to $5.0 \%$ (95\% UI 3.5, 6.7\%) in 2015. Countries like Burkina Faso (377.2\%), Liberia (270.8\%) and Chad (254.4\%) recorded the highest increases in the age-standardized proportion of deaths attributable to overweight/obesity in the last 25 years. Except for all the Southern SSA countries, Burundi and Comoros (Eastern SSA counties), all other nations recorded increases in diet-related deaths. Burkina Faso (64.7\%) and Liberia (48.7\%) had the highest increase in the age-standardized proportion of deaths attributable to dietary risks of NCD (Table 2 and online supplementary material, Supplemental Tables 6,7 and 8).

SSA achieved a significant reduction in the agestandardized proportion of deaths related to childhood undernutrition, discontinued breast-feeding and vitamin A deficiency. On the other hand, an increase of the proportion of deaths related to all components of dietary risks of NCD was recorded (Table 3).

The age-standardized proportion of deaths caused by CMNN diseases related to CMU fell significantly in all subregions of SSA. On the other hand, the proportion of NCD deaths attributable to dietary risk factors and overweight/ obesity either increased or remained relatively stable (Table 2 ).

\section{Discussion}

In the present study we found that the mortality burden attributable to CMU, overweight/obesity and dietary risks 


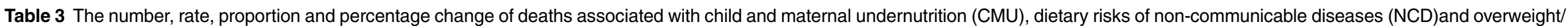
obesity in sub-Saharan Africa between 1990 and 2015

\begin{tabular}{|c|c|c|c|c|c|c|c|c|c|c|c|c|c|c|c|c|c|c|}
\hline \multirow[b]{3}{*}{ Risk factor } & \multicolumn{8}{|c|}{ Unstandardized (2015) } & \multicolumn{10}{|c|}{ Age-standardized (1990 and 2015) } \\
\hline & \multirow[b]{2}{*}{ Number } & \multirow[b]{2}{*}{$95 \%$ UI } & \multirow[b]{2}{*}{$\begin{array}{l}\text { Rate (per } \\
100000)\end{array}$} & \multirow[b]{2}{*}{$\begin{array}{l}95 \% \\
\mathrm{UI}\end{array}$} & \multirow{2}{*}{$\begin{array}{l}\text { Proportion } \\
(\%), \text { all- } \\
\text { cause† }\end{array}$} & \multirow[b]{2}{*}{$95 \%$ UI } & \multirow[b]{2}{*}{$\begin{array}{l}\text { Proportion (\%), } \\
\text { CMNND or CND } \ddagger\end{array}$} & \multirow[b]{2}{*}{$95 \%$ UI } & \multicolumn{4}{|c|}{ Rate (per 100 000) } & \multicolumn{6}{|c|}{ Proportion (\%), all-cause† } \\
\hline & & & & & & & & & 1990 & $95 \%$ UI & 2015 & $95 \%$ UI & 1990 & $95 \%$ UI & 2015 & $95 \%$ UI & $\begin{array}{c}\text { Change } \\
(\%)\end{array}$ & $95 \%$ UI \\
\hline $\begin{array}{l}\text { CMU } \\
\text { Childhood } \\
\text { undernutrition }\end{array}$ & $\begin{array}{r}826204 \\
730679\end{array}$ & $\begin{array}{l}737346,923789 \\
647336,823453\end{array}$ & $\begin{array}{l}86 \\
76\end{array}$ & $\begin{array}{l}77,96 \\
67,86\end{array}$ & $\begin{array}{r}10.3 \\
9.1\end{array}$ & $\begin{array}{l}9.1,11 \cdot 6 \\
8 \cdot 0,10 \cdot 4\end{array}$ & $\begin{array}{l}17.7 \\
15.7\end{array}$ & $\begin{array}{l}15 \cdot 7,19.9 \\
13 \cdot 8,17.7\end{array}$ & $\begin{array}{l}156 \\
135\end{array}$ & $\begin{array}{l}138,174 \\
117,152\end{array}$ & $\begin{array}{l}53 \\
41\end{array}$ & $\begin{array}{l}47,59 \\
36,46\end{array}$ & $\begin{array}{l}8.5 \\
7.4\end{array}$ & $\begin{array}{l}7.4,9.5 \\
6.3,8.4\end{array}$ & $\begin{array}{l}3 \cdot 8^{*} \\
2 \cdot 9^{*}\end{array}$ & $\begin{array}{l}3.4,4 \cdot 3 \\
2 \cdot 5,3 \cdot 4\end{array}$ & $\begin{array}{l}-55.2 \\
-60.0\end{array}$ & $\begin{array}{l}-61 \cdot 1,-49.2 \\
-66.0,-53 \cdot 6\end{array}$ \\
\hline Childhood stunting & 136455 & 62418,236262 & 14 & 7,25 & 1.7 & $0.8,2.9$ & $2 \cdot 9$ & $1 \cdot 4,5 \cdot 1$ & 32 & 17,53 & 8 & 3,13 & 1.8 & $0.9,2 \cdot 9$ & $0.6^{*}$ & $0.3,0.9$ & -69.1 & $-79 \cdot 7,-58 \cdot 3$ \\
\hline $\begin{array}{l}\text { Childhood } \\
\text { underweight }\end{array}$ & 243432 & 194332,304245 & 25 & 20,32 & $3 \cdot 0$ & $2 \cdot 4,3.8$ & $5 \cdot 2$ & $4 \cdot 2,6 \cdot 5$ & 53 & 41,70 & 14 & 11,17 & $2 \cdot 9$ & $2 \cdot 2,3 \cdot 8$ & $1 \cdot 0^{*}$ & $0.8,1.2$ & $-66 \cdot 2$ & $-74 \cdot 5,-54 \cdot 2$ \\
\hline Childhood wasting & 679606 & 572543,776337 & 71 & 60,81 & 8.5 & $7 \cdot 1,9 \cdot 8$ & 14.6 & $12 \cdot 2,16 \cdot 7$ & 118 & 97,141 & 38 & 32,43 & 6.4 & $5 \cdot 2,7 \cdot 8$ & $2 \cdot 7^{*}$ & $2 \cdot 3,3 \cdot 2$ & -57.4 & $-65 \cdot 2,-48 \cdot 2$ \\
\hline $\begin{array}{l}\text { Suboptimal breast- } \\
\text { feeding }\end{array}$ & 198263 & 131082,275781 & 21 & 14,29 & 2.5 & $1.6,3.5$ & 4.3 & $2 \cdot 8,6 \cdot 0$ & 28 & 19,38 & 11 & 7,15 & 1.5 & $1 \cdot 0,2 \cdot 1$ & 0.8 & $0.5,1.1$ & -48.5 & $-59 \cdot 0,-35 \cdot 9$ \\
\hline $\begin{array}{l}\text { Non-exclusive breast- } \\
\text { feeding }\end{array}$ & 181045 & 115278,255182 & 19 & 12,27 & $2 \cdot 3$ & $1 \cdot 5,3 \cdot 2$ & 3.9 & $2 \cdot 5,5 \cdot 5$ & 25 & 16,34 & 10 & 6,14 & 1.3 & $0.9,1.9$ & 0.7 & $0.5,1 \cdot 0$ & $-47 \cdot 2$ & $-58 \cdot 1,-34 \cdot 1$ \\
\hline $\begin{array}{l}\text { Discontinued breast- } \\
\text { feeding }\end{array}$ & 21899 & 7028, 42927 & 2 & 1,4 & 0.3 & $0.1,0.5$ & 0.5 & $0.2,0.9$ & 4 & 1,8 & 1 & 0,2 & 0.2 & $0.1,0.4$ & 0.1 & $0.0,0.2$ & -58 & $-69 \cdot 2,-43 \cdot 0$ \\
\hline $\begin{array}{l}\text { Vitamin A deficiency } \\
\text { Zn deficiency } \\
\text { Fe deficiency }\end{array}$ & $\begin{array}{l}47350 \\
33626 \\
62060\end{array}$ & $\begin{array}{r}27913,69775 \\
1951,74941 \\
44679,84503\end{array}$ & $\begin{array}{l}5 \\
4 \\
6\end{array}$ & $\begin{array}{l}3,7 \\
0,8 \\
5,9\end{array}$ & $\begin{array}{l}0.6 \\
0.4 \\
0.8\end{array}$ & $\begin{array}{l}0.3,0.9 \\
0.0,0.9 \\
0.6,1.0\end{array}$ & $\begin{array}{l}1.0 \\
0.7 \\
1.3\end{array}$ & $\begin{array}{l}0.6,1.5 \\
0.0,1.6 \\
1 \cdot 0,1.8\end{array}$ & $\begin{array}{r}19 \\
7 \\
16\end{array}$ & $\begin{array}{r}11,30 \\
0,15 \\
12,20\end{array}$ & $\begin{array}{r}3 \\
2 \\
10\end{array}$ & $\begin{array}{l}2,4 \\
0,4 \\
8,14\end{array}$ & $\begin{array}{l}1.0 \\
0.4 \\
0.9\end{array}$ & $\begin{array}{l}0.6,1.6 \\
0.0,0.9 \\
0.6,1.1\end{array}$ & $\begin{array}{l}0.2^{\star} \\
0.1 \\
0.7\end{array}$ & $\begin{array}{l}0.1,0.3 \\
0.0,0.3 \\
0.6,1.0\end{array}$ & $\begin{array}{l}-81.3 \\
-62.8 \\
-12.4\end{array}$ & $\begin{array}{l}-87 \cdot 3,-73 \cdot 4 \\
-72 \cdot 7,-48 \cdot 1 \\
-23 \cdot 6,4 \cdot 0\end{array}$ \\
\hline $\begin{array}{l}\text { Fe deficiency } \\
\text { Dietary risks }\end{array}$ & 558578 & $\begin{array}{r}446 / 9,84503 \\
453433,680197\end{array}$ & $\begin{array}{l}6 \\
58\end{array}$ & $\begin{array}{r}5,9 \\
47,71\end{array}$ & $\begin{array}{l}0.8 \\
7.0\end{array}$ & $\begin{array}{l}0.6,1 \cdot 0 \\
5 \cdot 8,8 \cdot 3\end{array}$ & 20.5 & $\begin{array}{l}17.0,1 \cdot 8 \\
17 \cdot 3,24 \cdot 3\end{array}$ & $\begin{array}{r}16 \\
191\end{array}$ & $\begin{array}{l}12,20 \\
158,231\end{array}$ & $\begin{array}{r}10 \\
169\end{array}$ & $\begin{array}{l}\text { 8, } 14 \\
138,208\end{array}$ & $\begin{array}{r}0.9 \\
10.4\end{array}$ & $\begin{array}{l}0.7,12 \\
8 \cdot 7,12.6\end{array}$ & $\begin{array}{r}0.7 \\
12.2\end{array}$ & $\begin{array}{c}0.6,1.0 \\
10 \cdot 2,14.7\end{array}$ & $\begin{array}{r}-12.4 \\
17.2\end{array}$ & $\begin{array}{r}-23 \cdot 6,4 \cdot 0 \\
11.9,22.5\end{array}$ \\
\hline Diet low in fruits & 173675 & 113390,242591 & 18 & 12,25 & 2.2 & $1.5,2.9$ & 6.4 & $4.3,8.6$ & 57 & 39,78 & 49 & 32,69 & $3 \cdot 1$ & $2 \cdot 1,4 \cdot 2$ & 3.5 & $2 \cdot 4,4.9$ & 13.5 & $7 \cdot 2,20 \cdot 0$ \\
\hline $\begin{array}{l}\text { Diet low in whole } \\
\text { grains }\end{array}$ & 133971 & 85649,191670 & 14 & 9,20 & 1.7 & $1 \cdot 1,2 \cdot 4$ & 4.9 & $3 \cdot 2,7 \cdot 0$ & 43 & 27,61 & 37 & 24,54 & $2 \cdot 3$ & $1 \cdot 5,3 \cdot 3$ & $2 \cdot 7$ & $1 \cdot 7,3 \cdot 9$ & $16 \cdot 1$ & $10 \cdot 1,21 \cdot 8$ \\
\hline Diet low in vegetables & 123544 & 67075,190913 & 13 & 7,20 & 1.5 & $0 \cdot 8,2 \cdot 4$ & 4.5 & $2 \cdot 5,6 \cdot 9$ & 43 & 23,66 & 36 & 20,56 & $2 \cdot 3$ & $1 \cdot 3,3 \cdot 6$ & $2 \cdot 6$ & $1 \cdot 4,4 \cdot 0$ & $13 \cdot 1$ & $6 \cdot 4,18 \cdot 7$ \\
\hline $\begin{array}{l}\text { Diet low in nuts and } \\
\text { seeds }\end{array}$ & 102661 & 64118,147118 & 11 & 7,15 & 1.3 & $0.8,1 \cdot 8$ & 3.8 & & 34 & 22,49 & 32 & 20,45 & 1.9 & $1 \cdot 2,2 \cdot 6$ & $2 \cdot 3$ & $1 \cdot 4,3 \cdot 2$ & 21.9 & $15 \cdot 9,28 \cdot 3$ \\
\hline Diet high in $\mathrm{Na}$ & 101044 & 25201,238911 & 11 & 3,25 & 1.3 & $0 \cdot 3,3 \cdot 0$ & 3.7 & $0 \cdot 9,8 \cdot 8$ & 35 & 9,89 & 31 & 8,77 & 1.9 & $0 \cdot 5,4 \cdot 8$ & $2 \cdot 2$ & $0.6,5.5$ & $16 \cdot 4$ & $4 \cdot 3,43 \cdot 7$ \\
\hline $\begin{array}{l}\text { Diet low in seafood } \\
n-3 \text { fatty acids }\end{array}$ & 72605 & 31205,118470 & 8 & 3,12 & 0.9 & $0.4,1.5$ & $2 \cdot 7$ & $1 \cdot 2,4 \cdot 3$ & 25 & 11,40 & 22 & 9,36 & 1.3 & $0 \cdot 6,2 \cdot 1$ & 1.6 & $0.7,2 \cdot 6$ & $17 \cdot 6$ & $10 \cdot 0,24 \cdot 5$ \\
\hline $\begin{array}{l}\text { Diet high in processed } \\
\text { meat }\end{array}$ & 33854 & 14548,54713 & 4 & 2,6 & 0.4 & $0.2,0.7$ & $1 \cdot 2$ & $0 \cdot 6,2 \cdot 0$ & 10 & 4,16 & 10 & 4,16 & 0.6 & $0.2,0.9$ & 0.7 & $0 \cdot 3,1 \cdot 1$ & $29 \cdot 0$ & $20 \cdot 1,47 \cdot 8$ \\
\hline $\begin{array}{l}\text { Diet high in trans-fatty } \\
\text { acids }\end{array}$ & 16679 & 5192, 35015 & 2 & 1,4 & 0.2 & $0.1,0.4$ & 0.6 & $0 \cdot 2,1 \cdot 3$ & 5 & 2,11 & 5 & 1,10 & 0.3 & $0.1,0.6$ & 0.3 & $0.1,0.7$ & $15 \cdot 0$ & $5 \cdot 2,23 \cdot 5$ \\
\hline $\begin{array}{l}\text { Diet high in sugar- } \\
\text { sweetened } \\
\text { beverages }\end{array}$ & $\begin{array}{l}8120 \\
2939\end{array}$ & 2059,4102 & 0 & 0,0 & $\begin{array}{l}0.1 \\
0.0\end{array}$ & $\begin{array}{l}.1,0.1 \\
0.0,0.1\end{array}$ & 0.1 & $\begin{array}{l}\cdot .2,0.4 \\
0.1,0.2\end{array}$ & 1 & $\begin{array}{l}2,3 \\
0,1\end{array}$ & 1 & $\begin{array}{l}2,3 \\
0,1\end{array}$ & $\begin{array}{l}.1 \\
0.0\end{array}$ & $\begin{array}{l}0.1,0.2 \\
0.0,0.1\end{array}$ & 0.1 & $\begin{array}{l}.1,0.2 \\
0.0,0.1\end{array}$ & $\begin{array}{l}32.8 \\
37.1\end{array}$ & $23 \cdot 2,52 \cdot 3$ \\
\hline Diet low in fibre & 4537 & 1414,9404 & 0 & 0,1 & 0.1 & $0.0,0.1$ & 0.2 & $0.1,0.4$ & 2 & 1,3 & 1 & 0,3 & 0.1 & $0 \cdot 0,0 \cdot 2$ & 0.1 & $0.0,0.2$ & $17 \cdot 4$ & $5 \cdot 8,29 \cdot 0$ \\
\hline Diet low in milk & 5729 & 1986, 9624 & 1 & 0,1 & 0.1 & $0.0,0.1$ & 0.2 & $0.1,0.4$ & 2 & 1,3 & 2 & 1,3 & 0.1 & $0.0,0.2$ & 0.1 & $0.0,0.2$ & 33.9 & $25 \cdot 0,43.0$ \\
\hline Diet high in red meat & 1734 & 474,3116 & 0 & 0,0 & 0.0 & $0.0,0.0$ & 0.1 & $0.0,0.1$ & 0 & 0,1 & 0 & 0,1 & 0.0 & $0.0,0.0$ & 0.0 & $0.0,0.1$ & 46.8 & $29 \cdot 7,63.8$ \\
\hline Overweight/obesity & 266768 & 189051,353096 & 28 & 20,37 & 3.3 & $2 \cdot 4,4 \cdot 4$ & 9.8 & $7 \cdot 0,12 \cdot 8$ & 59 & 39, 83 & 73 & 51,97 & 3.2 & $2 \cdot 1,4 \cdot 6$ & $5 \cdot 3$ & $3.8,7.0$ & 63.3 & $47 \cdot 9,83 \cdot 5$ \\
\hline
\end{tabular}

$\mathrm{UI}$, uncertainty interval; CMNND, communicable, maternal, neonatal and nutritional diseases.

The sum of numbers, rates and percentages in rows exceeds the total because of overlap between various risk factors. $0 \%$ represents very low proportion

Bold font indicates level 2 or level 3 categories of the risk factors.

*Significantly increased or decreased based on $95 \%$ UI.

toportions are for all causes of death.

fProportions are for specific category of diseases (i.e. CMNND accounting for CMU; NCD accounting for dietary risks and overweight/obesity). 


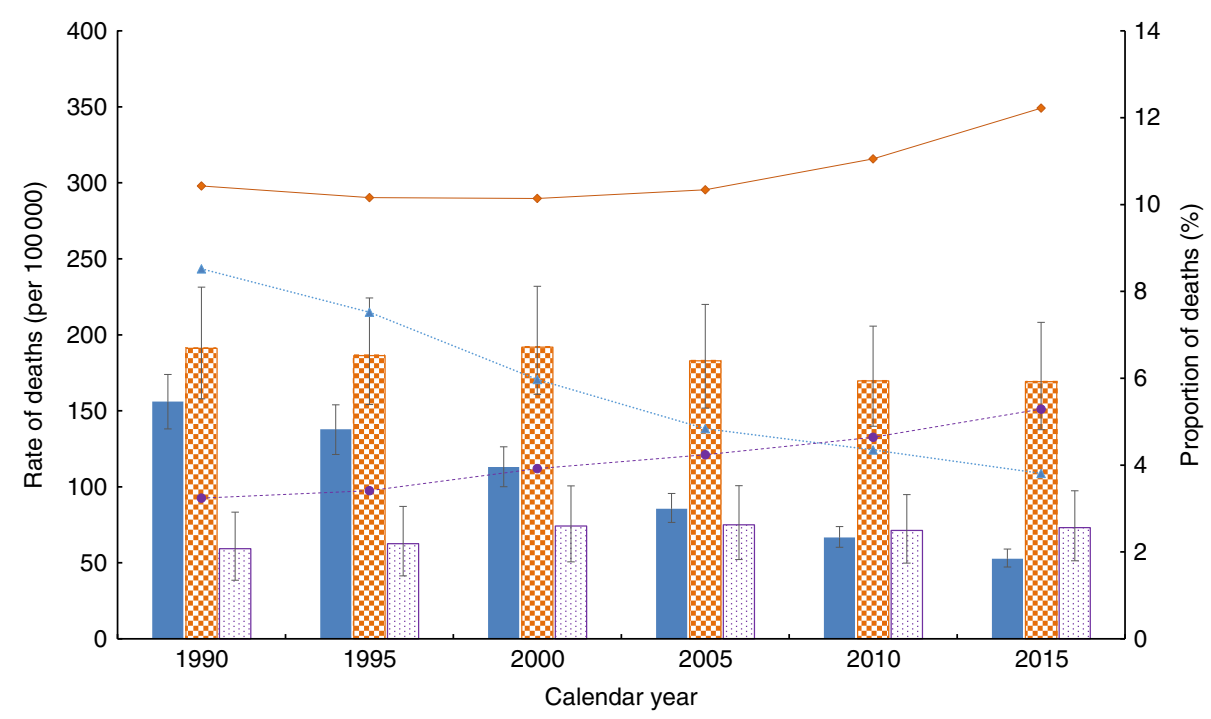

Fig. 3 (colour online) Age-standardized rate and proportion of deaths (all-cause) associated with child and maternal undernutrition (CMU), overweight/obesity and dietary risks of non-communicable diseases (NCD) in sub-Saharan countries between 1990 and

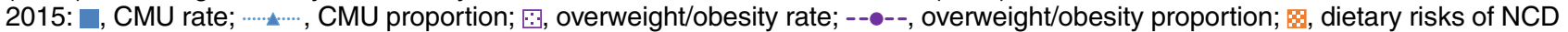
rate; $\multimap$, dietary risks of NCD proportion. Rates are mean values with their $95 \%$ uncertainty intervals represented by vertical bars

of NCD in SSA is high, indicating that the burden of diseases in the region is related to complex behavioural and metabolic risk factors. The relative contribution of dietary risks of NCD to all-cause deaths was significantly higher compared with overweight/obesity and CMU. Deaths attributable to CMU have decreased significantly in the past 25 years and similar patterns were found at subregional level. Each of the SSA countries has shown different levels of increase or decrease in mortality attributable to the CMU, overweight/obesity and dietary risk factors of NCD. To the best our knowledge, our study is the first of its kind to investigate the number (proportion) and trend of mortality attributable to a double burden of malnutrition (i.e. mortality attributable to CMU (eight risk factors), overweight/obesity and dietary risks of NCD (fourteen risk factors)) in the SSA, SSA sub-regions and countries. In addition, our results provide additional insights by strengthening the body of evidence on the existence of a double burden of nutrition-related diseases, which has not been given full attention by stakeholders. The findings could inform and highlight to governments and stakeholders in SSA countries the need to design and implement appropriate interventions to tackle this unprecedented phenomenon.

\section{The paradox}

Our findings show the coexistence of a significant burden of mortality attributable to CMU, overweight/obesity and dietary risk factors in SSA. In 2015, CMU and dietary risks of NCD were the second (behind unsafe sex) and third most important behavioural risk factors for mortality ${ }^{(30)}$. Overweight/obesity was also the second most important metabolic risk of mortality in SSA (behind systolic blood pressure $)^{(2,30)}$. In line with our findings, studies highlighted that the burden of NCD attributable to dietary risks is substantially high in addition to the already existing burden of undernutrition ${ }^{(8)}$. Furthermore, evidence also shows that the prevalence of obesity in Africa is high and increasing ${ }^{(5,11)}$. The coexistence of a high burden of both under- and overnutrition in SSA is also reflected at country level. For instance, in rural Nigeria, the prevalence of overweight (BMI $\left.=0-29.9 \mathrm{~kg} / \mathrm{m}^{2}\right) /$ obesity $\left(\right.$ BMI $\left.\geq 30.0 \mathrm{~kg} / \mathrm{m}^{2}\right)$ in adults and elderly people and of underweight (weightfor-age $Z$-score $<-2$ ) in school-aged children was found to be $30 \cdot 0^{(31)}$ and $70 \cdot 5 \%{ }^{(32)}$, respectively. Zeba et al. ${ }^{(33)}$ reported that $23.5 \%$ of adults in Burkina Faso had at least one CVD risk factor and one nutritional deficiency. In addition to socio-economic and technological developments, urbanization ${ }^{(34)}$ and an increase in per capita income $^{(35)}$, the minimal policy responses to the growing burden of NCD could explain the high burden of dietrelated diseases in $\mathrm{SSA}^{(36)}$. Increases in agricultural production and changes in trade policies (by increasing the availability and accessibility of processed foods) were also found to be drivers of the problem ${ }^{(37)}$. Given the multifaceted factors of nutrition-related burden of diseases in SSA, multisectoral collaboration at the macro and micro levels is a key to addressing the problem effectively.

Another important finding of the present study is the paradoxical trend in mortality attributable to CMU, overweight/obesity and dietary risks of NCD over the past 25 years. The mortality related to overweight/obesity and dietary risks of NCD has increased significantly, despite the deaths attributable to CMU still remaining substantially high. This finding mirrors studies that have shown the substantial contribution of both infectious diseases and NCD to the current burden in SSA - highlighting the 
non-classical epidemiological transition ${ }^{(38)}$. The burden of CMNN diseases has also persisted despite the emerging burden of $\mathrm{NCD}^{(39)}$. In line with other studies ${ }^{(8)}$, our findings suggest that SSA is undergoing a nutrition transition. However, the coexistence of a significant burden of disease associated with both over- and undernutrition shows that the transition is atypical ${ }^{(8)}$. Many hypotheses have been postulated to explain this pattern. A study by Popkin and Gordon-Larsen ${ }^{(40)}$ explained that such a change is associated with a faster increase of diet- and obesityrelated NCD compared with the socio-economic developments that SSA countries have achieved.

From the epigenetic point of view, studies also indicate that those who have exposure to diets with low energy and poor micronutrient contents in their early life are more likely to develop chronic diseases when they are challenged with high-energy foods and a sedentary life ${ }^{(41-43)}$, resulting in an 'NCD epidemic'. Evidence shows that this could happen as a result of genetic susceptibility to NCD via fetal programming ${ }^{(4,45)}$ and thrifty growth $^{(46,47)}$.

Global development agendas and initiatives have also impacted the current trend. For instance, the World Food Summit and the Millennium Development Goals largely ignored NCD and their risk factors. However, NCD were identified as a major health challenge for the Sustainable Development Goals, which have the objective of reducing premature mortality due to NCD by one-third ${ }^{(48)}$. As a result, countries should identify effective policy options and responses to prevent and control the increasing burden of NCD and the risk factors in SSA ${ }^{(49)}$. In this regard, despite the fact that a number of SSA countries have already formulated NCD policy frameworks, existing challenges, including limited implementation capacity, competing public health problems and the lack of effective and feasible means to integrate policies into the existing health-care system, have precluded the translation of these frameworks into practice ${ }^{(50)}$. The governments of SSA countries should recognize the importance of reorienting public health strategies and health-care services to provide the ability to address both child and maternal health problems and NCD.

\section{Dietary risks of non-communicable diseases}

Mortality either increased or remained the same for specific components of dietary risks of NCD over the past 25 years. Diets low in fruit, vegetables, whole grains, seeds and nuts, and high in $\mathrm{Na}$ were the most important dietary risk factors. In SSA and low-income countries, consumption of fruits and vegetables is very low ${ }^{(51,52)}$. In Ethiopia, Mozambique and South Africa, for instance, consumption of the recommended level of fruit and vegetables was reported to be very minimal ${ }^{(53-55)}$. It is also found that $3.2 \%$ of total deaths in South Africa are associated with low intake of fruits and vegetables ${ }^{(56)}$. In low-income countries, low consumption of fruits and vegetables is highly correlated with affordability ${ }^{(52)}$. In addition, a recent analysis of the Global Dietary Database showed that consumption of whole grains, nuts and seeds in SSA has decreased in the past two decades ${ }^{(51)}$. Na intake was also found to be high in some of the SSA countries. For instance, $81.0 \%$ of the adult population in SSA consumes more than the WHO recommendation level $(2 \mathrm{~g} / \mathrm{d})^{(57)}$.

Recognizing malnutrition as a major global health, economic and sustainable development challenge, the UN aims to improve overall nutrition through the Decade of Action on Nutrition $(2016-2025)^{(58)}$. This programme addresses all forms of malnutrition, from stunting, wasting and micronutrient deficiencies to overweight, obesity and nutrition-related NCD. The programme gives a unique opportunity for SSA countries and stakeholders to scale up their interventions against the double burden of malnutrition. As part of these interventions, actions to increase knowledge and awareness of communities on diet and nutrition should be supported. Effective strategies to promote healthy dietary habits should be designed using the experiences of other countries ${ }^{(59)}$. Promoting nutritionsensitive agriculture and value chain supply should be part of the strategies. Improving access to and use of appropriate information and communication technologies could also help in disseminating messages related to dietary behaviour and body weight, as well as in monitoring the effectiveness of interventions ${ }^{(60,61)}$. Evidence also shows that electronic and mobile health (e- \& mHealth) interventions are effective in promoting healthy diet-related behaviours in developing countries ${ }^{(62)}$.

\section{Regional variation}

The burden levels of CMU, overweight/obesity and dietary risk factors vary across sub-regions and countries. The highest age-standardized proportion of deaths attributable to CMU was found in Chad. In 2012, UNICEF reported that the proportion of children with acute malnutrition (wasting) in Chad was significantly higher than the WHO's emergency threshold $(15 \%)^{(63)}$. On the other hand, the highest age-standardized proportion of deaths attributable to overweight/obesity and dietary risks of NCD was found in South Africa (11.6\%) and Cameroon (17.8\%), respectively. In line with this, studies in these countries reported a high prevalence of overweight, obesity and NCD in the adult population, showing that the countries are at latter stages of nutritional transition ${ }^{(64,65)}$. International trade institutions such as an increasing number of processed food companies, and urbanization and consequent increase in the consumption of poor-quality diet, play a significant role in the burden of $\mathrm{NCD}^{(64,66,67)}$. SSA countries should develop and implement a strong and sustainable health information system to tackle the growing burden of NCD and their risk factors. Countries should also devise multisectoral interventions, including tailored and integrated agricultural, nutrition and public health policies, to overcome the 
nutrition-related burden of diseases. Countries and international organizations should recognize the effect of globalization on diet-related NCD burden ${ }^{(37)}$ and develop strategies to prevent and mitigate the impact.

\section{Limitations}

Although the GBD 2015 study used robust methods to estimate the burden of disease related to risk factors, limitations are inevitable. These limitations are discussed in detail elsewhere ${ }^{(1,2,28)}$; however, some of the most important limitations specific to the present study are discussed as follows. First, the relative risks were obtained from meta-regression analysis and used across the countries. Despite this, different risk factors could have different levels of effects across population subgroups. Second, availability of limited data in the region, particularly on dietary risk factors, could affect the estimates by over- or underestimating the burden. Third, because the urban and rural settings are substantially different environments in SSA countries, the estimates might not be applicable to these population subgroups separately. Fourth, nutritional risk factors were measured using different approaches. For instance, $\mathrm{Zn}$ deficiency was measured using a proxy exposure. Exposure to $\mathrm{Zn}$ deficiency (as an average total absorbed $\mathrm{Zn}$ ) was determined based on the ratio of $\mathrm{Zn}$ to phytate in foods. Lastly, publication bias could affect the results, compromising the comparability of burden of diseases related to risk factors. For instance, more published data are available on childhood undernutrition than diet-related NCD in $\mathrm{SSA}^{(2)}$.

\section{Conclusion}

In summary, whereas mortality attributable to undernutrition is declining significantly, the burden related to overnutrition is increasing. We found that the burden of mortality attributable to both under- and overnutrition in SSA is high, which highlights the presence of a double burden of malnutrition. SSA countries are in an important time of challenges and opportunities to overcome nutrition-related (both under- and overnutrition) burden of disease before the impact worsens. Evidence-based interventions and programmes should be designed to tackle this phenomenon in SSA. Tailored public health and nutritional policies should be designed to address the paradox. Multisectoral collaboration at different levels of the region is a key to further reduce the burden of diseases associated with undernutrition and to tackle the oncoming burden of overnutrition. Further prospective investigations, including biomarker and genetic studies, should be conducted to understand the specific factors of the complex feature of this unprecedented nutrition transition in the SSA countries.

\section{Acknowledgements}

Acknowledgements: The analyses in this article used available data from the GBD study 2015. The authors are grateful to the GBD team in the Institute for Health Metrics and Evaluation (IHME) at the University of Washington for availing the data. Y.A.M. is grateful for the support provided by an Australian Government Research Training Programme Scholarship. Financial support: The GBD study 2015 was supported by the Bill \& Melinda Gates Foundation. The current analysis received no specific grant from any funding agency in the public, commercial or not-for-profit sectors. Conflict of interest: All authors declare that they have no competing interests. The authors are solely responsible for the views expressed in this article, and they do not necessarily represent the views, decisions or policies of their institutions. Authorship: Y.A.M. conceived the study. Y.A.M. extracted and compiled the data and wrote all drafts of the manuscript. All authors provided data, assisted the data interpretation, critically reviewed the manuscript for intellectual input, and approved the final version submitted for publication. Ethics of buman subject participation: Not applicable.

\section{Supplementary material}

To view supplementary material for this article, please visit https://doi.org/10.1017/S1368980018002975

\section{References}

1. Forouzanfar MH, Alexander L, Anderson HR et al. (2015) Global, regional, and national comparative risk assessment of 79 behavioural, environmental and occupational, and metabolic risks or clusters of risks in 188 countries, 19902013: a systematic analysis for the Global Burden of Disease Study 2013. Lancet 386, 2287-2323.

2. Forouzanfar MH, Afshin A, Alexander LT et al. (2016) Global, regional, and national comparative risk assessment of 79 behavioural, environmental and occupational, and metabolic risks or clusters of risks, 1990-2015: a systematic analysis for the Global Burden of Disease Study 2015. Lancet 388, 1659-1724.

3. Taniguchi K \& Wang X (2003) Nutrition Intake and Economic Growth: Studies on the Cost of Hunger. Rome: FAO.

4. Vos T, Barber RM, Bell B et al. (2015) Global, regional, and national incidence, prevalence, and years lived with disability for 301 acute and chronic diseases and injuries in 188 countries, 1990-2013: a systematic analysis for the Global Burden of Disease Study 2013. Lancet 386, 743-800.

5. Kengne AP, Echouffo-Tcheugui J-B, Sobngwi E et al. (2013) New insights on diabetes mellitus and obesity in Africa Part 1: prevalence, pathogenesis and comorbidities. Heart 99, 979-983.

6. Ezzati M \& Riboli E (2013) Behavioral and dietary risk factors for noncommunicable diseases. N Engl J Med 369, 954-964.

7. Negin J, Cumming R, de Ramirez SS et al. (2011) Risk factors for non-communicable diseases among older adults in rural Africa. Trop Med Int Health 16, 640-646. 
8. Vorster HH, Kruger A \& Margetts BM (2011) The nutrition transition in Africa: can it be steered into a more positive direction? Nutrients 3, 429-441.

9. Chan M (2011) Noncommunicable Diseases Damage Health, Including Economic Health. New York: WHO.

10. Prentice AM (2006) The emerging epidemic of obesity in developing countries. Int J Epidemiol 35, 93-99.

11. Afshin A, Forouzanfar MH, Reitsma MB et al. (2017) Health effects of overweight and obesity in 195 countries over 25 years. $N$ Engl J Med 377, 13-27.

12. Wamani H, Åstrøm AN, Peterson S et al. (2007) Boys are more stunted than girls in sub-Saharan Africa: a metaanalysis of 16 demographic and health surveys. BMC Pediatr 7, 1.

13. World Health Organization (2017) The Double Burden of Malnutrition. Policy Brief. Geneva: WHO; available at http://apps.who.int/iris/bitstream/10665/255413/1/WHONMH-NHD-17.3-eng.pdf

14. Abrahams Z, Mchiza Z \& Steyn NP (2011) Diet and mortality rates in sub-Saharan Africa: stages in the nutrition transition. BMC Public Health 11, 1.

15. Kosaka S \& Umezaki M (2017) A systematic review of the prevalence and predictors of the double burden of malnutrition within households. Br J Nutr 117, 1118-1127.

16. Wojcicki JM (2014) The double burden household in subSaharan Africa: maternal overweight and obesity and childhood undernutrition from the year 2000: results from World Health Organization Data (WHO) and Demographic Health Surveys (DHS). BMC Public Health 14, 1124.

17. Jones AD, Acharya Y \& Galway LP (2016) Urbanicity gradients are associated with the household- and individuallevel double burden of malnutrition in sub-Saharan Africa. $J$ Nutr 146, 1257-1267.

18. Byass P, de Savigny D \& Lopez AD (2014) Essential evidence for guiding health system priorities and policies: anticipating epidemiological transition in Africa. Glob Health Action 7, 23359.

19. Global Burden of Disease Study 2015 (2016) Global Burden of Disease Study 2015 (GBD 2015) Results by Location, Cause, and Risk Factor. Seattle, WA: Institute for Health Metrics and Evaluation.

20. Murray CJ \& Lopez AD (1997) Global mortality, disability, and the contribution of risk factors: Global Burden of Disease Study. Lancet Glob Health 349, 1436-1442.

21. Wang H, Naghavi M, Allen C et al. (2016) Global, regional, and national life expectancy, all-cause mortality, and causespecific mortality for 249 causes of death, 1980-2015: a systematic analysis for the Global Burden of Disease Study 2015. Lancet 388, 1459-1544.

22. World Cancer Research Fund \& American Institute for Cancer Research (2007) Food, Nutrition, Physical Activity, and the Prevention of Cancer: A Global Perspective. Washington, DC: AICR.

23. Vos T, Allen C, Arora M et al. (2016) Global, regional, and national incidence, prevalence, and years lived with disability for 310 diseases and injuries, 1990-2015: a systematic analysis for the Global Burden of Disease Study 2015. Lancet 388, 1545-1602.

24. Ng M, Fleming T, Robinson M et al. (2014) Global, regional, and national prevalence of overweight and obesity in children and adults during 1980-2013: a systematic analysis for the Global Burden of Disease Study 2013. Lancet 384, 766-781.

25. Rasmussen CE \& Williams CKI (2006) Gaussian Processes for Machine Learning. Cambridge, MA: The MIT Press.

26. Flaxman AD, Vos T, Murray CJL et al. (2015) An Integrative Metaregression Framework for Descriptive Epidemiology, 1st ed. Seattle, WA: University of Washington Press.

27. Barendregt JJ, van Oortmarssen GJ, Vos T et al. (2003) A generic model for the assessment of disease epidemiology: the computational basis of DisMod II. Popul Health Metr 1, 4 .
28. Melaku YA, Temesgen AM, Deribew A et al. (2016) The impact of dietary risk factors on the burden of noncommunicable diseases in Ethiopia: findings from the Global Burden of Disease study 2013. Int J Behav Nutr Phys Act 13, 122.

29. Rubinstein RY \& Kroese DP (1966) Simulation and the Monte Carlo Method, 2nd ed, Hoboken, NJ: WileyInterscience.

30. Institute for Health Metrics and Evaluation (2015) GBD Compare. http://vizhub.healthdata.org/gbd-compare (accessed October 2016).

31. Ejim EC, Okafor CI, Emehel A et al. (2011) Prevalence of cardiovascular risk factors in the middle-aged and elderly population of a Nigerian rural community. JTrop Med 2011, 308687.

32. Oninla SO, Owa JA, Onayade AA et al. (2007) Comparative study of nutritional status of urban and rural Nigerian school children. J Trop Pediatr 53, 39-43.

33. Zeba AN, Delisle HF, Renier G et al. (2012) The double burden of malnutrition and cardiometabolic risk widens the gender and socio-economic health gap: a study among adults in Burkina Faso (West Africa). Public Health Nutr 15, 2210-2219.

34. Delisle H, Ntandou-Bouzitou G, Agueh V et al. (2011) Urbanisation, nutrition transition and cardiometabolic risk: the Benin study. Br J Nutr 107, 1534-1544.

35. Popkin BM (2015) Nutrition transition and the global diabetes epidemic. Curr Diab Rep 15, 64.

36. Lachat C, Otchere S, Roberfroid D et al. (2013) Diet and physical activity for the prevention of noncommunicable diseases in low- and middle-income countries: a systematic policy review. PLoS Med 10, e1001465.

37. Hawkes C (2006) Uneven dietary development: linking the policies and processes of globalization with the nutrition transition, obesity and diet-related chronic diseases. Global Health $2,4$.

38. Agyei-Mensah S \& de-Graft Aikins A (2010) Epidemiological transition and the double burden of disease in Accra, Ghana. J Urban Health 87, 879-897.

39. Murray CJL, Barber RM, Foreman KJ et al. (2015) Global, regional, and national disability-adjusted life years (DALYs) for 306 diseases and injuries and healthy life expectancy (HALE) for 188 countries, 1990-2013: quantifying the epidemiological transition. Lancet 386, 2145-2191.

40. Popkin BM \& Gordon-Larsen P (2004) The nutrition transition: worldwide obesity dynamics and their determinants. Int J Obes Relat Metab Disord 28, Suppl. 3, S2-S9.

41. Schwarz PE, Towers GW, van der Merwe A et al. (2009) Global meta-analysis of the $\mathrm{C}-11377 \mathrm{G}$ alteration in the ADIPOQ gene indicates the presence of population-specific effects: challenge for global health initiatives. Pharmacogenomics J 9, 42-48.

42. Olckers A, Towers GW, van der Merwe A et al. (2007) Protective effect against type 2 diabetes mellitus identified within the ACDC gene in a black South African diabetic cohort. Metabolism 56, 587-592.

43. Victora CG, Adair L, Fall C et al. (2008) Maternal and child undernutrition: consequences for adult health and human capital. Lancet 371, 340-357.

44. Canani RB, Di Costanzo M, Leone L et al. (2011) Epigenetic mechanisms elicited by nutrition in early life. Nutr Res Rev 24, 198-205.

45. Waterland RA \& Jirtle RL (2004) Early nutrition, epigenetic changes at transposons and imprinted genes, and enhanced susceptibility to adult chronic diseases. Nutrition 20, 63-68.

46. Lelijveld N, Seal A, Wells JC et al. (2016) Chronic disease outcomes after severe acute malnutrition in Malawian children (ChroSAM): a cohort study. Lancet Glob Health 4, e654-e662.

47. Dulloo AG, Jacquet J, Seydoux J et al. (2006) The thrifty 'catch-up fat' phenotype: its impact on insulin sensitivity 
during growth trajectories to obesity and metabolic syndrome. Int J Obes (Lond) 30, Suppl. 4, S23-S35.

48. United Nations (n.d.) Sustainable Development Goals Knowledge Platform. https://sustainabledevelopment.un. org/ (accessed December 2016).

49. Renzaho AMN (2015) The post-2015 development agenda for diabetes in sub-Saharan Africa: challenges and future directions. Glob Health Action 8, 27600.

50. Temu F, Leonhardt M, Carter J et al. (2014) Integration of non-communicable diseases in health care: tackling the double burden of disease in African settings. Pan Afr MedJ 18, 202.

51. Global Panel on Agriculture and Food Systems for Nutrition (2016) Food Systems and Diets: Facing the Challenges of the 21st Century. London: Global Panel on Agriculture and Food Systems for Nutrition.

52. Miller V, Yusuf S, Chow CK et al. (2016) Availability, affordability, and consumption of fruits and vegetables in 18 countries across income levels: findings from the Prospective Urban Rural Epidemiology (PURE) study. Lancet Glob Health 4, e695-e703.

53. Demissie T, Ali A \& Zerfu D (2009) Availability and consumption of fruits and vegetables in nine regions of Ethiopia with special emphasis to vitamin A deficiency. Ethiop $J$ Health Dev 23, 3.

54. Peltzer K \& Phaswana-Mafuya N (2012) Fruit and vegetable intake and associated factors in older adults in South Africa. Glob Health Action 5, 1-8.

55. Padrão P, Laszczyńska O, Silva-Matos C et al. (2011) Low fruit and vegetable consumption in Mozambique: results from a WHO STEPwise approach to chronic disease risk factor surveillance. Br J Nutr 107, 428-435.

56. Schneider M, Norman R, Steyn N et al. (2007) Estimating the burden of disease attributable to low fruit and vegetable intake in South Africa in 2000. S Afr Med J 97, $717-723$.

57. Oyebode O, Oti S, Chen Y-F et al. (2016) Salt intakes in subSaharan Africa: a systematic review and meta-regression. Popul Health Metr 14, 1.
58. World Health Organization (2016) United Nations Decades of Action on Nutrition. http://www.who.int/nutrition/dec ade-of-action/en/ (accessed January 2017).

59. Millett C, Laverty AA, Stylianou N et al. (2012) Impacts of a national strategy to reduce population salt intake in England: serial cross sectional study. PLoS One 7, e29836.

60. Afshin A, Babalola D, McLean M et al. (2016) Information technology and lifestyle: a systematic evaluation of internet and mobile interventions for improving diet, physical activity, obesity, tobacco, and alcohol use. J Am Heart Assoc 5, e003058.

61. Kerr DA, Harray AJ, Pollard CM et al. (2016) The connecting health and technology study: a 6-month randomized controlled trial to improve nutrition behaviours using a mobile food record and text messaging support in young adults. Int J Behav Nutr Phys Act 13, 52.

62. Müller AM, Alley S, Schoeppe S et al. (2016) The effectiveness of e- \& mHealth interventions to promote physical activity and healthy diets in developing countries: a systematic review. Int J Behav Nutr Phys Act 13, 109

63. UNICEF (2012) Survey results: Child malnutrition rates remain alarming in Chad. http://www.unicef.org/health/ chad_65674.html (accessed June 2016).

64. Steyn NP, Nel JH, Parker W et al. (2012) Urbanisation and the nutrition transition: a comparison of diet and weight status of South African and Kenyan women. Scand J Public Health 40, 229-238.

65. Echouffo-Tcheugui JB \& Kengne AP (2011) Chronic noncommunicable diseases in Cameroon - burden, determinants and current policies. Global Health 7, 44.

66. Powles J, Fahimi S, Micha R et al. (2013) Global, regional and national sodium intakes in 1990 and 2010: a systematic analysis of $24 \mathrm{~h}$ urinary sodium excretion and dietary surveys worldwide. BMJ Open 3, e003733.

67. Bosu WK (2015) An overview of the nutrition transition in West Africa: implications for non-communicable diseases. Proc Nutr Soc 74, 466-477. 